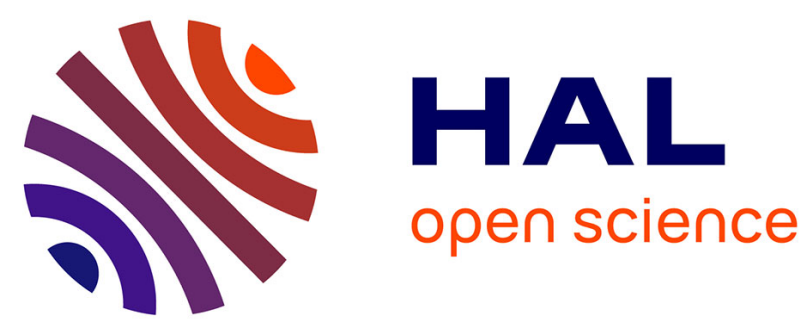

\title{
DiCoDiLe: Distributed Convolutional Dictionary Learning
}

Thomas Moreau, Alexandre Gramfort

\section{To cite this version:}

Thomas Moreau, Alexandre Gramfort. DiCoDiLe: Distributed Convolutional Dictionary Learning. IEEE Transactions on Pattern Analysis and Machine Intelligence, In press. hal-02371715v2

\section{HAL Id: hal-02371715 \\ https://hal.science/hal-02371715v2}

Submitted on 24 Nov 2020

HAL is a multi-disciplinary open access archive for the deposit and dissemination of scientific research documents, whether they are published or not. The documents may come from teaching and research institutions in France or abroad, or from public or private research centers.
L'archive ouverte pluridisciplinaire HAL, est destinée au dépôt et à la diffusion de documents scientifiques de niveau recherche, publiés ou non, émanant des établissements d'enseignement et de recherche français ou étrangers, des laboratoires publics ou privés. 


\title{
DiCoDiLe: Distributed Convolutional Dictionary Learning
}

\author{
Thomas Moreau and Alexandre Gramfort
}

\begin{abstract}
Convolutional dictionary learning (CDL) estimates shift invariant basis adapted to represent signals or images. CDL has proven useful for image denoising or inpainting, as well as for pattern discovery on multivariate signals. Contrarily to standard patch-based dictionary learning, patterns estimated by CDL can be positioned anywhere in signals or images. Optimization techniques consequently face the difficulty of working with extremely large inputs with millions of pixels or time samples. To address this optimization problem, we propose a distributed and asynchronous algorithm, employing locally greedy coordinate descent and a soft-locking mechanism that does not require a central server. Computation can be distributed on a number of workers which scales linearly with the size of the data. The parallel computation accelerates the parameter estimation and the distributed setting allows our algorithm to be used with data that do not fit into a single computer's RAM. Experiments confirm the theoretical scaling properties of the algorithm. This allows to demonstrate an improved pattern recovery as images grow in size, and to learn patterns on images from the Hubble Space Telescope containing tens of millions of pixels.
\end{abstract}

Index Terms - Convolutional Dictionary Learning, Distributed System, Coordinate Descent.

\section{INTRODUCTION}

Cor ONVOLUTIONAL sparse linear models consider that signals or images are obtained by linear combination of a few patterns localized in time or space. Patterns, which typically have a finite support, encode local variations such as edges in images or transient events in signals. The benefit of this model over traditional sparse coding technique based on local patches is that it is shift-invariant. While sparse coding encodes every local patch in the data, the convolutional linear model selects and encodes few patches leading to sparser and sharper reconstruction [3]. Over the last ten to fifteen years, these models have been used successfully for various signal and image processing applications. It was first proposed by Grosse et al. [14] for music recognition. It was then used extensively for denoising and inpainting of images [5, 17, 24, 34]. Beyond denoising or inpainting, recent applications used this shiftinvariant model as a way to learn and localize relevant patterns in signals or images. Yellin et al. [35] used it to count the number of blood-cells present in holographic lens-free images. Jas et al. [15] and Dupré la Tour et al. [10] learned recurring patterns in univariate and multivariate signals from neuroscience. This model has also been used for denoising astronomical data [29] and localizing predefined patterns from space satellite images [9]. For both denoising and pattern recovery tasks, there is a clear advantage in using large images. It is an expected statistical effect that when more realisations of the patterns are present, it is easier to disentangle them from other patterns and from the noise. Thus, for all aforementioned applications, there is a need for scalable and efficient algorithm that can handle large images.

Convolutional dictionary learning (CDL) is the technique commonly employed to estimate the parameters of such shiftinvariant models. The dictionary is here formed by the set of patterns (or atoms), whose positions in time or space are encoded by some sparse activation vectors. CDL then boils down to an optimization problem, where the variables are the dictionary as well as the activations. This problem is nonconvex and approximate solutions can be found by using alternating minimization over each variable. The estimation of

- T. Moreau and A. Gramfort are with Université Paris-Saclay, Inria, CEA, Palaiseau, 91120, France. the activation - which is commonly referred to as convolutional sparse coding (CSC) - is the critical bottleneck when it comes to applying CDL to large data.

As CSC is a convex problem, classical optimization tools can be employed. Chalasani et al. [6] used an accelerated proximal gradient method based on Fast Iterative Soft-Thresholding Algorithm (FISTA; [2]). Bristow et al. [5] proposed the Fast Convolutional Sparse Coding (FCSC) method, a splitting algorithm based on Alternating Direction Method of Multipliers (ADMM; [12]). While it is possible to leverage the Fast Fourier Transform to reduce the complexity of these approaches [34], both algorithms require to compute Fourier transforms of the entire data at each iteration. The cost can therefore be prohibitive for large signals or images. To avoid such costly global operations, several works have proposed to use local computations [14, 17, 21, 24, 37]. A first line of work proposed by Kavukcuoglu et al. [17] uses greedy coordinate descent (GCD; [23]) with an efficient mechanism to compute the update value. In their seminal work, Grosse et al. [14] propose to adapt the Feature Sign Search algorithm to only process small window of the signal at a time. Similarly, Papyan et al. [24] proposed to solve the problem locally over smaller continuous chunks of signal and then aggregate these local results by solving a global optimization problem. In our recent work [21], we proposed to use locally greedy coordinate descent (LGCD). Based on updates similar to GCD, this method has a lower periteration complexity as it relies on local computation in the data. It does so like a patch-based technique would do, yet solving the non-local problem.

To further reduce the computational burden of CDL, recent studies consider parallelization strategies. Skau and Wohlberg [28] proposed a parallel method based on consensus ADMM. It does so by splitting the computation across the different atoms. This limits the number of cores that can be used to the number of atoms in the dictionary, and it still requires Fourier transforms on the entire data. Concurrently, we proposed an alternative parallel algorithm for CSC, called DICOD [21], that distributes GCD updates when working with one dimensional signals. DICOD could be used with multidimensional data such as images, yet only by splitting the data along one dimension, hence limiting its interest in this setting. 
In the present paper, we propose a distributed solver for $\mathrm{CDL}$, which can be used to learn recurring patterns in large multidimensional data. For the CSC step, we extend DICOD to use local updates with lower complexity while still working in the asynchronous setting without a central server. We detail a soft-lock mechanism, necessary to ensure the convergence of the algorithm with a grid of workers while remaining asynchronous. Then, we explain how to also leverage the distributed set of workers to further reduce the cost of the dictionary update. This is done by precomputing in parallel sufficient statistics used in the gradient operator. Extensive numerical experiments confirm the agreement between the theoretical complexity of our algorithm and its running time, allowing us to learn patterns from an astronomical image formed by tens of millions of pixels. A fully asynchronous implementation based on MPI and non-blocking communication primitives is made available to replicate our results.

In Section 2, we describe the convolutional linear model and the estimation of its parameters with CDL. Our distributed algorithm DiCoDiLe is introduced in Section 3. Section 4 presents results on simulations, signals and images, demonstrating improvements in both scalability and pattern recovery.

Notation For a vector $U \in \mathbb{R}^{P}$ we denote $U_{p} \in \mathbb{R}$ its $p^{\text {th }}$ coordinate. For a finite d-dimensional domain $\Omega=\prod_{i=1}^{d}\left[0, T_{i}[\right.$, where $\left[0, T_{i}\right.$ [ are the integers from 0 to $T_{i}-1,|\Omega|=\prod_{i=1}^{d} T_{i}$ is its size. We will denote $T=\prod_{i=1}^{d} T_{i}$ whenever the link between $T$ and $T_{i}$ is unambiguous. We denote $\mathcal{X}_{\Omega}^{P}$ (resp. $\mathcal{X}_{\Omega}^{P \times Q}$ ) the space of observations defined on $\Omega$ with values in $\mathbb{R}^{P}$ (resp. $\mathbb{R}^{P \times Q}$ ). For instance, $\mathcal{X}_{\Omega}^{3}$ with $d=2$ is the space of RGB-images with height $T_{1}$ and width $T_{2}$. The value of an observation $X \in \mathcal{X}_{\Omega}^{P}$ at position $\omega \in \Omega$ is given by $X[\omega]=X\left[\omega_{1}, \ldots, \omega_{d}\right] \in \mathbb{R}^{P}$, and its restriction to the $p^{t h}$ coordinate at each position is denoted $X_{p} \in \mathcal{X}_{\Omega}^{1}$. All signals are 0-padded, i.e. for $\omega \notin \Omega, X[\omega]=0$. The convolution operator is denoted $*$ and is defined for $X \in \mathcal{X}_{\Omega}^{P}$ and $Y \in \mathcal{X}_{\Omega}^{P \times Q}$ as the signal $X * Y \in \mathcal{X}_{\Omega}^{Q}$ with

$$
(X * \boldsymbol{Y})[\omega]=\sum_{\tau \in \Omega} X[\omega-\tau] \cdot \boldsymbol{Y}[\tau], \forall \omega \in \Omega
$$

For any signal $X \in \mathcal{X}_{\Omega}^{P}$, the reversed signal is defined as $X^{\uparrow}[\omega]=X\left[T_{1}-\omega_{1}, \ldots, T_{d}-\omega_{d}\right]^{\top}$. Computing the convolution with $X^{\uparrow}$ is equivalent to computing the cross-correlation with $X$. The $p$-norm is defined as $\|X\|_{p}=\left(\sum_{\omega \in \Omega}\|X[\omega]\|_{p}^{p}\right)^{1 / p}$. We denote $\mathcal{F}$ the Discrete Fourier transform (DFT) of a signal and for a multivariate signal $X \in \mathcal{X}_{\Omega}^{P}, \widehat{X}=\mathcal{F}(X)$ denotes the DFT of each of its coordinates. Finally, we will also denote ST the soft-thresholding operator defined as

$$
\mathrm{ST}(u, \lambda)=\operatorname{sign}(u) \max (|u|-\lambda, 0) .
$$

\section{Convolutional Dictionary Learning}

In this section, we recall the convolutional linear model and the classical way to estimate its parameters by alternating minimization.

\subsection{Convolutional Linear Model}

For an observed $X \in \mathcal{X}_{\Omega}^{P}$, signal or image, the convolutional linear model reads

$$
X=Z * D+\xi,
$$

with $D \in \mathcal{X}_{\Theta}^{K \times P}$ a dictionary of $K$ atoms $D_{k}$ in dimension $P$ defined on a sub-domain $\Theta=\prod_{i=1}^{d}\left[0, L_{i}[\subset \Omega\right.$, also called support of the atom. The activations are denoted $Z \in \mathcal{X}_{\Omega}^{K}$ and

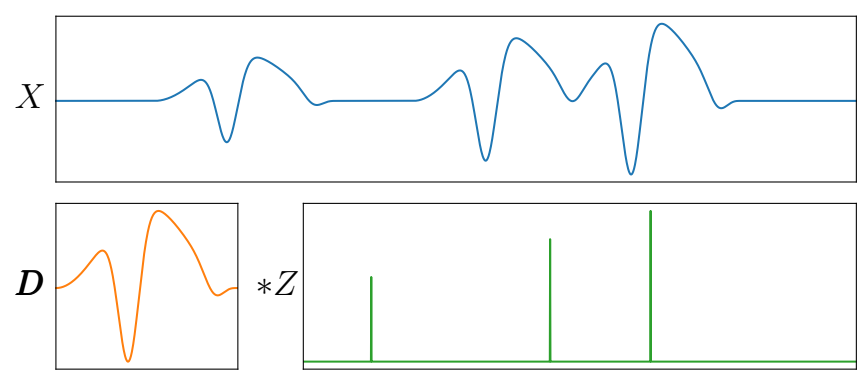

Fig. 1. Decomposition of a noiseless univariate signal $X$ (blue) as the convolution $Z * D$ between a temporal pattern $D$ (orange) and a sparse activation signal $Z$ (green).

$\xi \in \mathcal{X}_{\Omega}^{P}$ is an additive noise term which is typically considered white and Gaussian. The dictionary elements $\boldsymbol{D}_{k}$ are prototypical patterns present in the data. They typically have a much smaller support $\Theta$ than the full domain $\Omega$ (i.e. $L=|\Theta| \ll T$ ). The activations $Z_{k}$ encode the localization of the pattern $k$ in the data. A non-zero coefficient $Z_{k}[\omega]$ indicates the presence of the pattern $\boldsymbol{D}_{k}$ around the position $\omega$. As patterns typically occur at few locations in each observation, the coding signal $Z$ is considered to be sparse. The aim of this model is truly to separate the shape of the local variation in the observation encoded in the dictionary - from their localization in space or time. Figure 1 illustrates the decomposition of a temporal signal with one pattern.

The parameters of this model are estimated by solving the following CDL optimization problem:

$$
\min _{\substack{Z, \boldsymbol{D} \\\left\|\boldsymbol{D}_{k}\right\| \leq 1}} \underbrace{\frac{1}{2}\|X-Z * \boldsymbol{D}\|_{2}^{2}}_{F(Z, \boldsymbol{D})}+\underbrace{\lambda\|Z\|_{1}}_{G(Z)},
$$

where the first term $F(Z, D)$ measures how well the model fits the data and the second term $G(Z)$ promotes sparse activations. The regularization parameter $\lambda>0$ balances these two terms. The constraint $\left\|D_{k}\right\|_{2} \leq 1$ is used to avoid the scale ambiguity in our model due to the $\ell_{1}$ constraint. Indeed, the $\ell_{1}$-norm of $Z$ can be made arbitrarily small by rescaling $D$ by $\alpha \in \mathbb{R}_{+}^{*}$ and $Z$ by $\frac{1}{\alpha}$. This problem is bi-convex, i.e. it is not jointly convex in $(Z, D)$ but it is convex in each of its coordinates. It is usually solved with an alternating minimization algorithm, updating at each iteration one of the block of coordinates, $Z$ or $D$. As each of the sub-problems are convex, the alternated descent is guaranteed to decrease the cost function, converging to a critical point of (3). It is however not guaranteed to be a global minimizer.

\subsection{Convolutional Sparse Coding (CSC)}

Given a dictionary of patterns $D$, CSC aims to retrieve the sparse decomposition $Z^{*}$ associated to the signal $X$. When minimizing only over $Z$, (3) becomes

$$
Z^{\star}=\underset{Z}{\operatorname{argmin}} \frac{1}{2}\|X-Z * \boldsymbol{D}\|_{2}^{2}+\lambda\|Z\|_{1} .
$$

This boils down to a Lasso problem with a Toeplitz design matrix. Therefore, classical optimization techniques for the Lasso can be applied with the same convergence guarantees. In practice, these methods are adapted to leverage the convolutional structure of the problem, reducing the computational complexity to solve (4). 


\subsubsection{Locally Greedy Coordinate Descent (LGCD)}

Coordinate descent (CD) is an algorithm which updates a single coefficient at each iteration. Following Moreau et al. [21], we use locally greedy coordinate selection to solve efficiently the CSC (4). Algorithm 1 summarizes the computations for LGCD.

Coordinate update For (4), it is possible to compute efficiently the optimal update for a given coordinate when all the others are fixed. Denoting $q$ the iteration number, and $\widetilde{Z}_{k_{0}}^{(q)}\left[\omega_{0}\right]$ (atom $k_{0}$ at position $\left.\omega_{0}\right)$ the optimal value of the activation $Z_{k_{0}}^{(q)}\left[\omega_{0}\right]$, the update reads

$$
\widetilde{Z}_{k_{0}}^{(q)}\left[\omega_{0}\right]=\frac{1}{\left\|\boldsymbol{D}_{k_{0}}\right\|_{2}^{2}} \mathrm{ST}\left(\beta_{k_{0}}^{(q)}\left[\omega_{0}\right], \lambda\right)
$$

where $\beta^{(q)}$ is an auxiliary variable in $\mathcal{X}_{\Omega}^{K}$ defined for $(k, \omega) \in$ $[1, K] \times \Omega$ as

$$
\beta_{k}^{(q)}[\omega]=\left(\left(X-Z^{(q)} * \boldsymbol{D}+Z_{k}^{(q)}[\omega] e_{\omega} * \boldsymbol{D}_{k}\right) * \boldsymbol{D}_{k}^{\uparrow}\right)[\omega],
$$

and where $e_{\omega}$ is a dirac (canonical vector) with value 1 in $\omega$ and 0 elsewhere. The value of the auxillary variable in $\left(k_{0}, \omega_{0}\right)$ corresponds to the gradient of $F$ for a point $Z^{(q)}$ with the coordinate $\left(k_{0}, \omega_{0}\right)$ set to 0 . To enable efficient coordinate update, an important property is that $\widetilde{Z}^{(q)}[\omega]$ only depends on a small neighborhood around $\omega$.

Definition 2.1 ( $\Theta$-neighborhood). Let us consider a support $\Theta=$ $\prod_{i=1}^{d}\left[0, L_{i}\left[\right.\right.$. For $\Omega_{0} \in \Omega$, the $\Theta$-neighborhood $\mathcal{V}_{\Theta}\left(\omega_{0}\right)$ is defined as

$$
\mathcal{V}_{\Theta}\left(\omega_{0}\right)=\prod_{i=1}^{d}\left[\omega_{0, i}-L_{i}+1, \omega_{0, i}+L_{i}[\right.
$$

Proposition 2.2 (Weak dependence). If the coordinate $\left(k_{0}, \omega_{0}\right)$ is updated in $Z^{(q)}$, then the value of $\widetilde{Z}^{(q+1)}$ differs from $\widetilde{Z}^{(q)}$ only for $\omega$ in a $\Theta$-neighborhood $\mathcal{V}_{\Theta}\left(\omega_{0}\right)$ of $\omega_{0}$.

Proof. If the coordinate $Z_{k_{0}}\left[\omega_{0}\right]$ is updated with an additive update $\Delta Z_{k_{0}}\left[\omega_{0}\right]=\widetilde{Z}_{k_{0}}^{(q)}\left[\omega_{0}\right]-Z_{k_{0}}^{(q)}\left[\omega_{0}\right]$, Kavukcuoglu et al. [17] showed that $\beta^{(q+1)}$ can be obtained using the relation

$$
\beta_{k}^{(q+1)}[\omega]=\beta_{k}^{(q)}[\omega]-\left(\boldsymbol{D}_{k_{0}} * \boldsymbol{D}_{k}^{\dagger}\right)\left[\omega-\omega_{0}\right] \Delta Z_{k_{0}}^{(q)}\left[\omega_{0}\right],
$$

for all $(k, \omega) \neq\left(k_{0}, \omega_{0}\right)$. As the support of $\left(\boldsymbol{D}_{k_{0}} * \boldsymbol{D}_{k}^{\dagger}\right)[\omega]$ is $\mathcal{V}_{\Theta}(0)$, this implies that the value $\beta_{k}^{(q+1)}$ only changes in the neighborhood $\mathcal{V}_{\Theta}\left(\omega_{0}\right)=\omega_{0}+\mathcal{V}_{\Theta}(0)$.

This proposition shows that the optimal value for a coordinate only depends on the current values of the activations in a neighborhood of size $\left|\mathcal{V}_{\Theta}(0)\right|=2^{d} L$. This corresponds to twice the size of the dictionary in each dimension. Using this property, the computational complexity of updating a coordinate is $\mathcal{O}\left(2^{d} K L\right)$, as it is only necessary to update $\beta$ and $\widetilde{Z}^{(q)}$ on $\mathcal{V}_{\Theta}\left(\omega_{0}\right)$.

Coordinate selection The selection of the updated coordinate $\left(k_{0}^{(q)}, \omega_{0}^{(q)}\right)$ can follow different strategies. Following our work in $[10,21]$, we propose to select the coordinates with a locally greedy rule (LGCD).
Definition 2.3 (Disjoint and contiguous partition). Let us consider a domain $\Omega$. For $M \in \mathbb{N}$, a $M$ disjoint and contiguous partition $\left\{\mathcal{C}_{m}\right\}_{m=1}^{M}$ is a set of subsets of $\Omega$ such that

$$
\begin{aligned}
\prod_{i=1}^{d}\left[\omega_{i}, \omega_{i}^{\prime}\right] \subset \mathcal{C}_{m} \quad \text { for } \omega, \omega^{\prime} \in \mathcal{C}_{m}, \\
\bigcup_{m} \mathcal{C}_{m}=\Omega, \quad \mathcal{C}_{m} \cap \mathcal{C}_{m^{\prime}}=\emptyset \text { for } m \neq m^{\prime} .
\end{aligned}
$$

At iteration $q$, the coordinate to update is selected greedily among the $m$-th sub-domain $\mathcal{C}_{m}$ with

$$
\left(k_{0}^{(q)}, \omega_{0}^{(q)}\right)=\underset{(k, \omega) \in[1, K] \times \mathcal{C}_{m}}{\operatorname{argmax}}|\underbrace{\widetilde{Z}_{k}^{(q)}[\omega]-Z_{k}^{(q)}[\omega]}_{\Delta Z_{k}^{(q)}[\omega]}|,
$$

with $m=q \bmod M$. This strategy cycles through each of the $M$ sub-domains and select the coordinate in $\mathcal{C}_{m}$ which is the farthest away from its optimal value when all the other coordinates are fixed. The quantity $\Delta Z_{k}[\omega]$ acts as a proxy for the reduction of the cost function obtained with the update of coordinate $(k, \omega)$ to the value $\widetilde{Z}_{k}^{(q)}[\omega]$ [23]. The complexity of the locally greedy selection rule is linear with the size of the sub-segments $\mathcal{O}\left(K\left|\mathcal{C}_{m}\right|\right)$. In the case where $M=K T$, this rule boils down to the cyclic CD [11] with a coordinate selection complexity in $\mathcal{O}(1)$, while setting $M=1$ boils down to greedy $\mathrm{CD}$ [23] where the complexity of the selection is linear with the size of the signal $\mathcal{O}(K T)$. The selection of $M$ in LGCD acts as a tradeoff between the computational complexity and the convergence speed of these two methods.

As it was shown in recent studies [16, 22], updating in priority the important coordinates by greedy selection leads to faster convergence rates, although it increases the cost of one iteration. The LGCD strategy proposes to use sub-domains $\mathcal{C}_{m}$ such that the computational complexities of selecting and updating the coordinate match. By using sub-domains of the $\mathcal{V}_{\Theta}(0)^{\prime}$ s size i.e. $\left|\mathcal{C}_{m}\right|=2^{d} L$, the global iteration complexity of LGCD scales with $2^{d}$ times the size of the dictionary $D$ i.e. $\mathcal{O}\left(2^{d} K L\right)$. By doing so LGCD achieves faster convergence rates than nongreedy coordinate selection rule while limiting the per-iteration cost.

Stopping criterion The stopping criterion used for this algorithm follows [23]. The algorithm is stopped once the magnitude of all possible coordinate updates are below a threshold $\epsilon>0$ specified by the user, i.e.

$$
\max _{(k, \omega) \in[1, K] \times \Omega}\left|\Delta Z_{k}^{(q)}[\omega]\right|<\epsilon .
$$

\subsubsection{Global solvers}

Chalasani et al. [6] propose to solve (4) by proximal gradient methods exploiting the convolution in the gradient computation to save time. The core idea behind this method is to use a proximal splitting of the cost function between its smooth part $F(\cdot, \boldsymbol{D})$ and a proximable part $G$. Proximal gradient descent (ISTA) reads

$$
Z^{(q+1)}=\operatorname{prox}_{\gamma G}\left(Z^{(q+1)}-\gamma \nabla_{Z} F\left(Z^{(q+1)}, D\right)\right),
$$

where $\gamma$ is the step size of the algorithm, usually chosen as the inverse of the smoothness constant of $F$, and $\operatorname{prox}_{\gamma G}$ is the proximal operator of $\gamma G$. For $G(\cdot)=\lambda\|\cdot\|_{1}$, this corresponds to the soft-thresholding operator $\mathrm{ST}(\cdot, \gamma \lambda)$. To make this algorithm efficient the key is to rely on the FFT for the gradient computations, as described in [34]. Doing the computation in the 


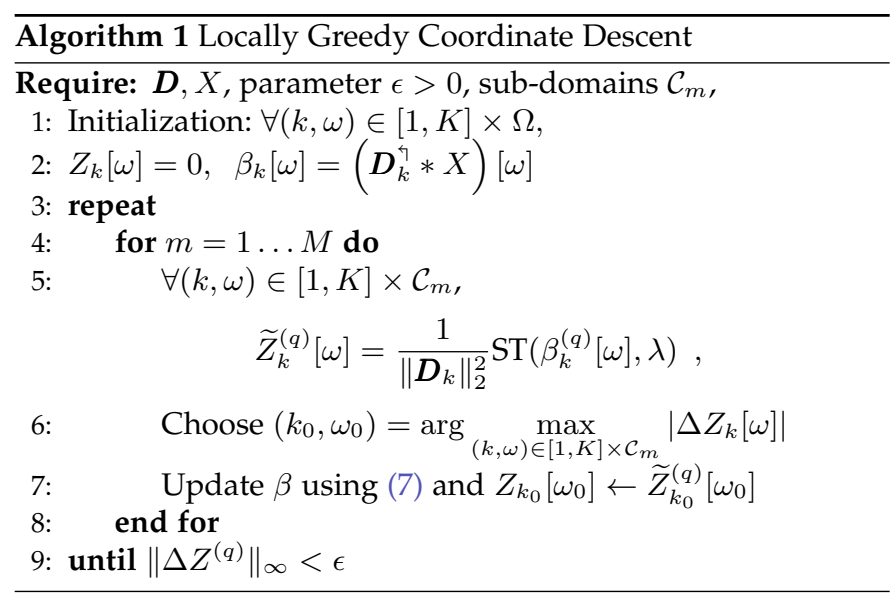

Fourier domain, the computational complexity of each iteration of this algorithm is $\mathcal{O}(K T \log T)$. It is also possible here to use an accelerated version FISTA to solve the problem $[2,6]$.

An alternative global algorithm proposed by Bristow et al. [5] is based on the ADMM algorithm [12]. The core idea in this line of work is to introduce an auxiliary variable and split the two part of the objective with an equivalent problem

$$
\min _{\widehat{Y}, Z} \frac{1}{2}\|\widehat{X}-\widehat{Y} \widehat{D}\|_{F}^{2}+\lambda\|Z\|_{1} \text { s.t. } \mathcal{F}(Z)=\widehat{Y} \text {. }
$$

This problem is then solved using ADMM in its scaled form [4, Section 3.1.1]. The critical part in this algorithm is the minimization of the augmented Lagrangian relatively to $Y$ which necessitates multiple FFT as well as the inversion of $T$ linear systems of size $K \times K$. The overall complexity of this method is thus $\mathcal{O}\left(K T\left(\log T+K^{2}\right)\right)$.

For these global solvers, the complexity of each iteration scales with the size of the data in $\mathcal{O}(T \log T)$ and the convergence rate is independent of $T$. In contrast, the iteration of LGCD are independent of $T$ but the number of iterations needed to reach convergence scales with the number of non-zero coefficients which also depends on $T$. For large and sparse data, LGCD tends to be more efficient as the overall complexity depends linearly in $T$ and not in $T \log T$. However, the choice between a local vs a global algorithm depends on the sparsity level of the desired solution, controlled by the value of the regularization parameter $\lambda[10]$.

\subsection{Dictionary Update}

Given an activation signal $Z^{(q)}$, the dictionary is updated to improve how the model reconstructs the signal. The problem reads

$$
\boldsymbol{D}^{*}=\underset{\left\|\boldsymbol{D}_{k}\right\|_{2} \leq 1}{\operatorname{argmin}} \frac{1}{2}\left\|X-Z^{(q)} * \boldsymbol{D}\right\|_{2}^{2} .
$$

This problem is smooth and convex and can be solved using algorithms designed for smooth constrained optimization.

\subsubsection{Projected Gradient Descent}

The projected gradient descent (PGD; [7]) and its accelerated version (APGD) are possible strategies. These two algorithms are equivalent to ISTA and FISTA iterations where the proximal operator is a simple projection [7]. The efficiency of these two methods relies on pre-computations of sufficient statistics for fast evaluation of the gradient of the objective $\nabla_{D} F$ [10]. The computations can be factorized as

$$
\nabla_{\boldsymbol{D}} F(Z, \boldsymbol{D})=Z^{\uparrow} *(X-Z * \boldsymbol{D})=\boldsymbol{\Psi}-\boldsymbol{\Phi} * \boldsymbol{D},
$$

where $\Phi \in \mathcal{X}_{\mathcal{V}_{\Theta}(0)}^{K \times K}$ and $\boldsymbol{\Psi} \in \mathcal{X}_{\Theta}^{K \times P}$ are respectively defined as the convolution $Z^{\natural} * Z$ restricted to $\mathcal{V}_{\Theta}(0)$ and the convolution $Z^{\natural} * X$ restricted to $\Theta$, i.e.

$$
\begin{aligned}
& \boldsymbol{\Phi}[\omega]=Z^{\dagger} * Z=\sum_{\tau \in \Omega} Z[\omega+\tau] Z^{\top}[\tau] \quad \text { for } \omega \in \mathcal{V}_{\Theta}(0), \\
& \boldsymbol{\Psi}[\omega]=Z^{\dagger} * X=\sum_{\tau \in \Omega} Z[\omega+\tau] X^{\top}[\tau] \quad \text { for } \omega \in \Theta .
\end{aligned}
$$

The complexity to obtain the two constants $\Phi$ and $\Psi$ scales as $\mathcal{O}(K(K+P) L T)$. This is comparable with the cost of computing the gradient directly $\mathcal{O}(K P T \log T)$. However, once the two constants are pre-computed, the gradient of $F$ is obtained using (13) with complexity $\mathcal{O}\left(2^{d} K^{2} P L \log (L)\right)$. This is independent of the data size $T$, making each iteration efficient.

\subsubsection{Related solvers}

The block coordinate descent for dictionary learning [20], which consists in updating one atom at a time, can also be extended to the convolutional case. As PGD for full dictionary updates, this technique benefits from sufficient statistics pre-computations and the complexity is similar. Another possibility is to rely on ADMM to update the dictionary $[5,34]$. However, ADMM algorithms can be slow to reach a convergence, yielding suboptimal reconstruction performances as the feasible point is not directly optimized but results from a consensus. Yellin et al. [35] proposed to adapt the K-SVD dictionary update method [1] to the convolutional setting but this technique is adapted to an $\ell_{0}$ penalty for the activation $Z$. It also requires to compute costly singular value decompositions (SVD).

\subsection{Choice of initialization and regularization parameter}

In practice, the quality of the solution obtained by CDL is impacted by the choice of the regularization parameter $\lambda$. Also, due to the iterative nature of the solvers, the initialization of the dictionary $\boldsymbol{D}^{0}$ can play an important role.

\section{Regularization parameter selection}

The choice of a regularization parameter $\lambda$ in the context of dictionary learning is often selected by cross-validation on a subsequent task such as denoising or classification. However in the context of purely unsupervised learning, e.g. matrix factorization, this is still an open problem. This problem has recently received some attention using Bayesian methods [25, 33]. For the specific case of CDL using an $\ell_{1}$ norm, it is useful to note that there exists a value $\lambda_{\max }$ such that for $\lambda \geq \lambda_{\max }, 0$ is solution of (4). The first order condition for (4) reads

$$
\nabla_{Z} F\left(0, D^{(0)}\right)=X * \boldsymbol{D}^{(0) \dagger} \in \partial G(0)=[-\lambda, \lambda]^{K T} .
$$

This condition is always verified if $\lambda>\lambda_{\max }=\left\|X * D^{(0)}\right\|_{\infty}$. In the following, the regularization parameter $\lambda$ is chosen as a fraction of $\lambda_{\max }$. Using this data dependent value, the ratio $\lambda / \lambda_{\max }$ is chosen in $[0,1]$, making the definition of the regularization parameter common to all input signals $X$.

\section{Dictionary initialization}

The standard strategy to initialize $\boldsymbol{D}^{(0)}$ for CDL methods is to generate random atoms with Gaussian white noise. However, as these atoms generally poorly correlate with the data, the initial value of $\lambda_{\max }$ is low compared to the following ones, leading to dense activation $Z$. For dictionary learning, a classical way to initialize the dictionary is to take samples from the input data [20]. In the convolutional setting, this boils down 


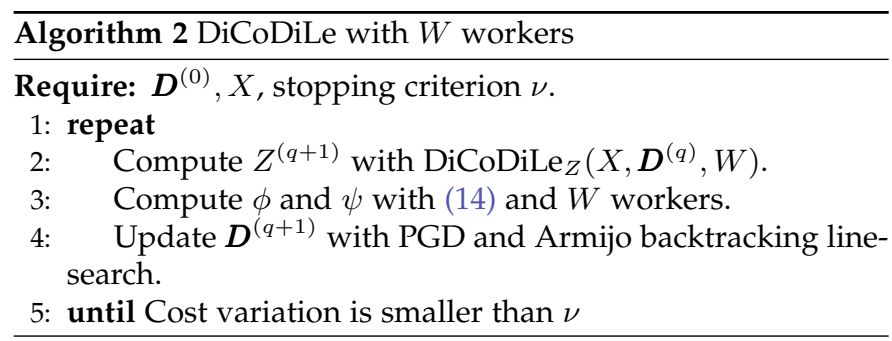

to taking random patches of the size of the dictionary directly from the data. This can be theoretically motivated by the work of Zhang et al. [36] that demonstrate improvements in pattern recovery under suitable assumptions.

\section{Distributed Convolutional Dictionary Learn- ING (DICODILE)}

In this section, we introduce DiCoDiLe, a distributed algorithm for convolutional sparse coding. Its steps are summarized in Algorithm 2. The CSC part of this algorithm will be referred to as DiCoDiLe $_{Z}$. It extends our previous work DICOD [21] by handling multidimensional data, in particular images. The algorithm proposes to distribute the computation on a grid of workers. To guarantee convergence with multidimensional data in an asynchronous setting, we introduce the notion of soft-locks described in Subsection 3.1. Details on how the dictionary updates can also benefit from the distributed setting are presented in Subsection 3.2.

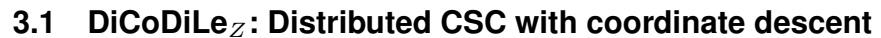

In the convolutional setting, coordinates that are far enough - relatively to the size of the dictionary - are only weakly dependent (Proposition 2.2). It is thus natural to distribute the computation of $\mathrm{CD}$ by splitting the data in continuous subdomains [21].

The $\operatorname{DiCoDiLe}_{Z}$ algorithm, described in Algorithm 3, is distributed over $W$ workers which update asynchronously the coordinates of $Z$. We consider $\left\{\mathcal{S}_{w}\right\}_{w=1}^{W}$, a disjoint and contiguous partition of $\Omega$. Each worker $w \in[1, W]$ is in charge of updating the coordinates of the sub-domain $\mathcal{S}_{w}$. These sub-domains are also partitioned with disjoint and contiguous sub-domains $\mathcal{C}_{m}^{(w)}$ of size $\left|\mathcal{V}_{\Theta}(0)\right|$. Then, the worker $w$ chooses at each iteration $q$ an update candidate $\left(k_{0}, \omega_{0}\right)$ which corresponds to a greedy update in one of its sub-domain $\mathcal{C}_{m}^{(w)}$, i.e.

$$
\left(k_{0}, \omega_{0}\right)=\underset{(k, \omega) \in[1, K] \times \mathcal{C}_{m}^{(w)}}{\operatorname{argmax}}\left|\Delta Z_{k}[\omega]\right|,
$$

with $m=q \bmod M$. The sub-domains $\mathcal{C}_{m}^{(w)}$ are selected in a cyclic order but empirical test showed no significant differences for random sub-domain selection. Then, this candidate update is accepted or rejected using the soft-lock mechanism described in Subsection 3.1.1. If the candidate is rejected, the worker moves on to the next sub-domain $\mathcal{C}_{m+1}^{w}$ without updating a coordinate. If the candidate is accepted, the value of the coordinate $Z_{k_{0}}\left[\omega_{0}\right]$ is replaced by $\widetilde{Z}_{k_{0}}^{(q)}\left[\omega_{0}\right]$ and $\beta$ is updated with (7). To ensure the convergence, it is sufficient to notify the neighboring workers if a local update changes the value of $\beta$ outside of $\mathcal{S}_{w}$, i.e. if $\mathcal{V}_{\Theta}\left(\omega_{0}\right) \not \subset \mathcal{S}_{w}$.

Definition $3.1\left(\Theta\right.$-Border). Let us consider a sub-domain, $\mathcal{S}_{w}=$ $\prod_{i=1}^{d}\left[l_{i}, u_{i}\left[\right.\right.$, where $\left(l_{i}, u_{i}\right)$ are the lower and upper limits of this

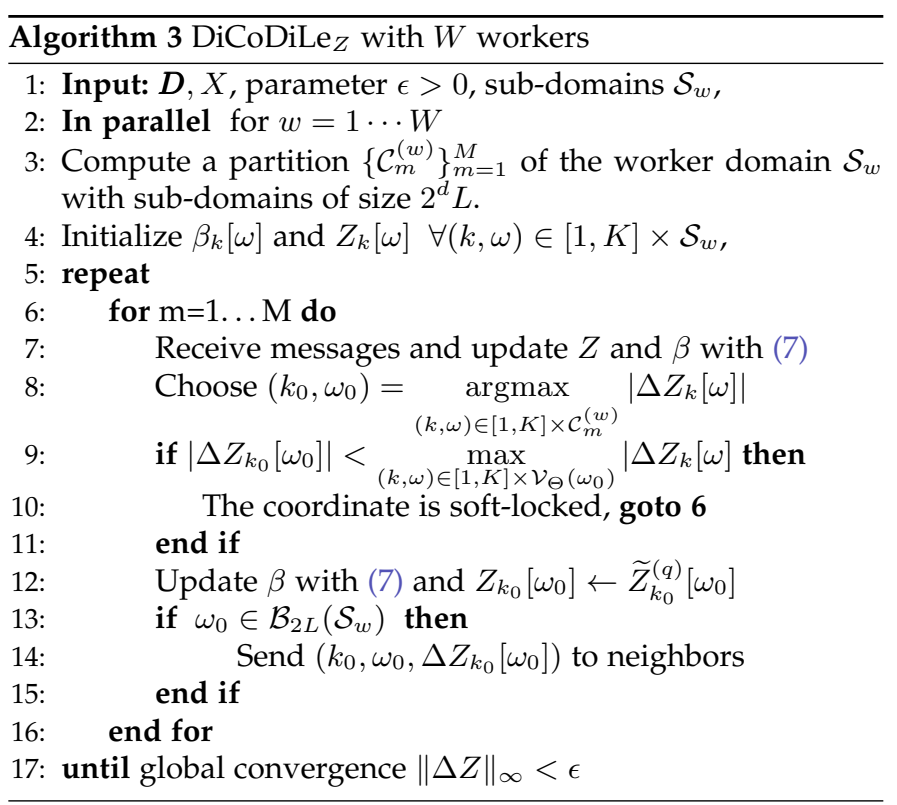

sub-domain for dimension $i$. For $\Theta=\prod_{i=1}^{d}\left[0, L_{i}[\right.$, the $\Theta$-border is defined as the set of points $\omega \in \mathcal{S}_{w}$ such that $\mathcal{V}_{\Theta}(\omega) \not \subset \mathcal{S}_{w}$, i.e.

$$
\mathcal{B}_{\Theta}\left(\mathcal{S}_{w}\right)=\prod_{i=1}^{d}\left[l_{i}, l_{i}+L_{i}\left[\cup \left[u_{i}-L_{i}, u_{i}[.\right.\right.\right.
$$

In the case where the updated coordinate $\left(k, \omega_{0}\right)$ is in $\mathcal{S}_{w}$ $\mathcal{B}_{\Theta}\left(\mathcal{S}_{w}\right)$, then $\mathcal{V}_{\Theta}\left(\omega_{0}\right)$ is included in the set of coordinates $\mathcal{S}_{w}$ updated by the worker $w$. In this case, the update is not at the border of the sub-domain, so the values of $\beta$ and $\widetilde{Z}^{(q)}$ need no update for the other workers $w^{\prime}$. Thus, there is no need to exchange information between the workers. However, for $\omega_{0} \in \mathcal{B}_{\Theta}\left(\mathcal{S}_{w}\right)$, it is necessary to notify other workers $w^{\prime}$ whose domain overlap with the $\Theta$-neighborhood of $\omega_{0}$ i.e. $\mathcal{V}_{\Theta}\left(\omega_{0}\right) \cap \mathcal{S}_{w^{\prime}} \neq \emptyset$. The notification can be done by sending the triplet $\left(k_{0}, \omega_{0}, \Delta Z_{k_{0}}\left[\omega_{0}\right]\right)$ to these workers. These information are sufficient to keep $\beta$ up to date for all workers using (7). Figure 2 illustrates this communication process on a $2 \mathrm{D}$ example. With this distributed algorithm, the inter-processes communications are minimal, as only $d+2$ values need to be exchanged. Moreover, as it does not rely on centralized communication, the necessary bandwidth between the workers is also reduced as a worker only communicates with its direct neighbors. This contrasts with other distributed algorithms for CDL such as [28] that requires centralized communication of full support values between the workers. Finally, the communication only occurs when $\omega_{0} \in \mathcal{B}_{\Theta}\left(\mathcal{S}_{w}\right)$, which happens rarely especially when working with large data and patterns of small support. This reduces even further the need of bandwidth between workers.

\subsubsection{Interferences and Soft-Locks in asynchronous setting}

While it is now clear how communication should be done between workers, one need to clarify how to proceed to ensure convergence in the case of asynchronous updates. When $W$ coordinates $\left(k_{w}, \omega_{w}\right)_{w=1}^{W}$ of $Z$ are updated simultaneously by respectively $\Delta Z_{k_{w}}\left[\omega_{w}\right]$, the updates might not be independent. The local version of $\beta$ used for the update does not account for the other updates. The cost difference resulting from all these simultaneous updates is denoted $\Delta E$, and the cost reduction induced by only one update $w$ is denoted $\Delta E_{k_{w}}\left[\omega_{w}\right]$. Simple 


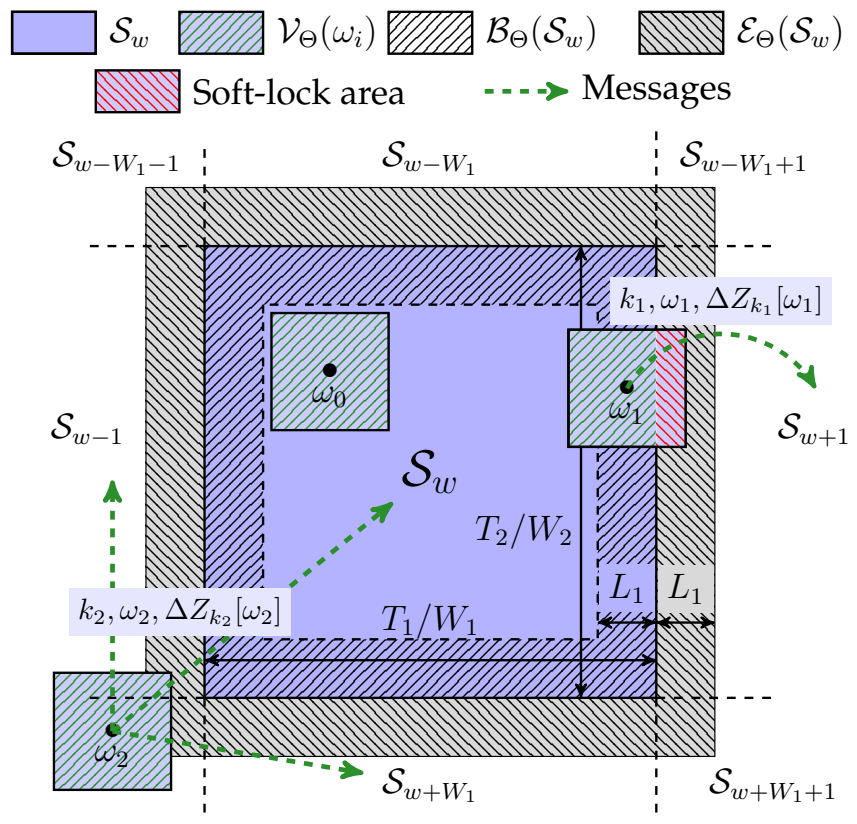

Fig. 2. Communication process in DiCoDiLe ${ }_{Z}$ with $d=2$ and 9 workers centered around worker $w$. The update in $\omega_{0}$ is independent of the other workers. The update in $\omega_{1}$ is performed if no better coordinate update is possible in the soft-lock area $\mathcal{V}_{\Theta}\left(\omega_{1}\right) \cap \mathcal{S}_{w+1}$ (red hatched area). If accepted, the worker $w+1$ needs to be notified. The update in $\omega_{2}$ changes the value of the optimal updates in the $\Theta$-extension of the other workers sub-domains. Thus it needs to notify all the neighboring workers.

derivations, detailed in Proposition A.2, show that

$$
\begin{aligned}
\Delta E & =\overbrace{\sum_{i=1}^{W} \Delta E_{k_{w}}\left[\omega_{w}\right]}^{\text {iterative steps }} \\
- & \underbrace{\sum_{w \neq w^{\prime}}\left(\boldsymbol{D}_{k_{w}} * \boldsymbol{D}_{k_{w^{\prime}}}^{\dagger}\right)\left[\omega_{w^{\prime}}-\omega_{w}\right] \Delta Z_{k_{w}}\left[\omega_{w}\right] \Delta Z_{k_{w^{\prime}}}\left[\omega_{w^{\prime}}\right]}_{\text {interference }})
\end{aligned}
$$

If for all $w$, all other updates $w^{\prime}$ are such that if $\omega_{w^{\prime}} \notin \mathcal{V}_{\Theta}\left(\omega_{w}\right)$, then $\left(\boldsymbol{D}_{k_{w}} * \boldsymbol{D}_{k_{w^{\prime}}}^{\uparrow}\right)\left[\omega_{w^{\prime}}-\omega_{w}\right]=0$. The updates can be considered to be sequential as the interference term is zero. This also directly results from Proposition 2.2. When $\left|\left\{\omega_{w}\right\}_{w} \cap \mathcal{V}_{\Theta}\left(\omega_{0}\right)\right|=$ $I_{0}>1$, the interference term does not vanish. Moreau et al. [21] show that if $I_{0}<3$, then, under mild assumption, the interference term can be controlled and DICOD converges. While this is sufficient for 1D partitioning as $I_{0}$ cannot be larger than 2 (if $\mathcal{S}_{w}$ is larger than $\mathcal{V}_{\Theta}(0)$ ), this analysis cannot be extended to $I_{0} \geq 3$. This limits the partitioning of $\Omega$ along one single dimension.

To avoid this limitation, we propose the soft-lock, a mechanism to avoid interfering updates of higher order. The idea is to avoid updating concurrent coordinates simultaneously. A classical tool in computer science to address such concurrency issue is to rely on a synchronization primitive, called lock. This primitive can ensure that at most one worker enters a protected block of code simultaneously. In our case, it would be possible to use this primitive to restrict updates in $\mathcal{B}_{\Theta}\left(\mathcal{S}_{w}\right)$ only when none of its neighbors is updating $\mathcal{B}_{\Theta}\left(\mathcal{S}_{w^{\prime}}\right)$. This would avoid interfering updates. However, the drawback of this method is that it prevents the algorithm to be asynchronous, as typical lock implementations rely on centralized communications. Instead, we propose to use an implicit locking mechanism which keeps our algorithm asynchronous.

Definition 3.2 ( $\Theta$-extension). We consider $\mathcal{S}_{w}=\prod_{i=1}^{d}\left[l_{i}, u_{i}[a\right.$ sub-domain of $\Omega$. For $\Theta=\prod_{i=1}^{d}\left[0, L_{i}\left[\right.\right.$, the $\Theta$-extension of $\mathcal{S}_{w}$ is defined as $\mathcal{E}_{\Theta}\left(\mathcal{S}_{w}\right)=\left(\mathcal{S}_{w}+\mathcal{V}_{\Theta}(0)\right) \backslash \mathcal{S}_{w}$, i.e.

$$
\mathcal{E}_{\Theta}\left(\mathcal{S}_{w}\right)=\prod_{i=1}^{d}\left[l_{i}-L_{i}, l_{i}\left[\cup \left[u_{i}, u_{i}+L_{i}[.\right.\right.\right.
$$

The idea of the soft-lock is the following. Additionally to the sub-domain $\mathcal{S}_{w}$, each worker also maintains the value of $\widetilde{Z}^{(q)}$ and $\beta$ on the $\Theta$-extension $\mathcal{E}_{\Theta}\left(\mathcal{S}_{w}\right)$ (See Figure 2). These extra coordinates are referred to as ghost cells in the computer science literature [18]. At each iteration, the worker $w$ considers a candidate coordinate $\left(k_{0}, \omega_{0}\right)$ to update from (17). If $\omega_{0} \notin \mathcal{B}_{\Theta}\left(\mathcal{S}_{w}\right)$, the coordinate is updated like in LGCD. When $\omega_{0} \in \mathcal{B}_{\Theta}\left(\mathcal{S}_{w}\right)$, the candidate is accepted if there is no better coordinate update in $\mathcal{V}_{\Theta}\left(\omega_{0}\right) \cap \mathcal{E}_{\Theta}\left(\mathcal{S}_{w}\right)$, i.e. if

$$
\left|\Delta Z_{k_{0}}\left[\omega_{0}\right]\right|>\max _{k, \omega \in[1, K] \times \mathcal{V}_{\Theta}\left(\omega_{0}\right) \cap \mathcal{E}_{\Theta}\left(\mathcal{S}_{w}\right)}\left|\Delta Z_{k}[\omega]\right| .
$$

In case of equality, the update in the sub-domain $\mathcal{S}_{w^{\prime}}$ with the minimal index $w^{\prime}$ is preferred. Using this mechanism effectively prevents any interfering update. If two workers $w<w^{\prime}$ have update candidates $\omega_{0}$ and $\omega_{0}^{\prime}$ such that $\omega_{0}^{\prime} \in \mathcal{V}_{\Theta}\left(\omega_{0}\right)$, then with (21), only one of the two updates will be accepted. It will be either the largest one if $\left|\Delta Z_{k_{0}}\left[\omega_{0}\right]\right| \neq\left|\Delta Z_{k_{0}^{\prime}}\left[\omega_{0}^{\prime}\right]\right|$ or the one from $w$ if both updates have the same magnitude. The other update will be rejected and there will be no interference. By doing so this mechanism is fully asynchronous. The updates on the borders are implicitly ordered, preventing interference. The update are guaranteed to be performed as if they were run sequentially, ensuring convergence of the algorithm. Unlike classical ghost cells [18], the soft-lock mechanism for DiCoDiLe does not require a synchronization step after each update in a worker. The update of the ghost cells in neighboring workers are handled using the asynchronous messages used to maintain $\beta$ up to date (see Algorithm 3 1.14). The soft-lock mechanism requires to increase slightly the communication between the workers and to double the size of the $\Theta$-border in each direction. This is however not a bottleneck as demonstrated in the following experiments.

\subsubsection{Speed-up analysis of DiCoDiLe ${ }_{Z}$}

In this section, we consider the speed-up provided by this distributed algorithm. As each worker runs LGCD locally, the computational complexity of a local iteration in a worker $w$ is $\mathcal{O}\left(2^{d} K L\right)$. This complexity is independent of the number of workers used to run the algorithm. Thus, the speed-up obtained is equal to the number of updates that are performed in parallel. If we consider $W$ update candidates $\left(k_{w}, \omega_{w}\right)$ chosen by the workers, the number of updates that are performed corresponds to the number of updates that are not soft-locked. To evaluate this, we make the assumption that the updates are uniformly distributed on each $\mathcal{S}_{w}$. In this case, the probability that an update candidate located in $\omega \in \mathcal{S}_{w}$ is not soft-locked can be lower bounded by

$$
P(\omega \notin \mathbf{S L}) \geq \prod_{i=1}^{d}\left(1-\frac{W_{i} L_{i}}{T_{i}}\right)
$$

Computations are detailed in Proposition B.1. When the ratios $\frac{T_{i}}{W_{i} L_{i}}$ are large for all $i$, i.e. when the size of the workers subdomains $\left|\mathcal{S}_{w}\right|$ are large compared to the dictionary size $L$, then $P(\omega \notin \mathbf{S L}) \simeq 1$. In this case, the expected number of accepted 
update candidates is close to $W$ and DiCoDiLe $z$ scales almost linearly. When $W_{i}$ reaches $\frac{T_{i}}{L_{i}}\left(\frac{2^{1 / d}}{2^{1 / d}-1}\right)$, then $P(\omega \notin \mathbf{S L}) \gtrsim \frac{1}{2}$, and the acceleration is reduced to $\frac{W}{2}$. Numerical experiments in Section 4 will evaluate if this result holds in practice, although the updates are not distributed uniformly when running LGCD on real data.

In comparison, we report in [21] a super-linear speed-up for DICOD on 1D signals. This is due to the fact that we are using GCD locally in each worker. GCD has a very high iteration complexity when $W$ is small, as it scales linearly with the size of the considered sub-domain. Thus, when sub-dividing the signal between more workers, the iteration complexity is decreasing and more updates are performed in parallel. This explains why the speed-up is almost quadratic for low $W$. As the complexity of iterative GCD are worse than LGCD, DiCoDiLe $z$ has better performance than DICOD in low $W$ regime. Furthermore, in the $1 \mathrm{D}$ setting, DICOD becomes similar to $\mathrm{DiCoDiLe}_{Z}$ once the size of the workers sub-domain becomes too small. This happens when the local domain of LGCD becomes comparable to the size of the sub-domains $\mathcal{S}_{w}$. This implies that $\operatorname{DiCoDiLe}_{Z}$ always outperforms DICOD.

\subsubsection{Stopping criterion}

In this distributed setting, it is non-trivial to know when the algorithm reaches convergence. To match the sequential stopping criterion of GCD (9), the convergence is considered to be reached once no worker can perform a coordinate update $\left|\Delta Z^{(q)}\right|$ that is higher than $\epsilon>0$. However, as a worker can be affected by its neighboring workers, the stopping criterion of CD cannot be applied independently in each worker. Once a worker reaches this state, the potential update can still increase above $\epsilon$ because of updates in $\mathcal{E}_{\Theta}\left(\mathcal{S}_{w}\right)$ by another worker. To make sure the correct criterion is achieved, workers that reach the local criterion are paused. They are restarted when a neighboring workers triggers an update on $\mathcal{E}_{\Theta}\left(\mathcal{S}_{w}\right)$. The global convergence can be decided when all workers are paused. While this process is based on centralized communication, it can still be performed asynchronously using message passing to a designated master process that will notify the end of the algorithm to all workers.

\subsection{Dictionary updates in a distributed setting}

For the dictionary, a change in a coordinate in $Z$ at any point in $\Omega$ can impact the solution of (12). Moreover, the atoms are interdependent of each other. It is thus complicated to parallelize the dictionary update as it is centralized by nature and there is no natural division of the computation. However, the size of $\boldsymbol{D}$ is way smaller than $Z$ and (12) can be solved efficiently when applying the PGD algorithm described in Subsection 2.3. The main computational bottleneck is the computation of the constants $\boldsymbol{\Phi}$ and $\boldsymbol{\Psi}$. For these, it is possible to leverage the distributed setting as the computation are separable between the different workers. The computations can be made embarrassingly parallel among the workers as

$$
\begin{array}{ll}
\boldsymbol{\Phi}[\omega]=\sum_{w=1}^{W} \sum_{\tau \in \mathcal{S}_{w}} Z[\tau] Z^{\top}[\tau+\omega] \quad \text { for } \omega \in \mathcal{V}_{\Theta}(0), \\
\boldsymbol{\Psi}[\omega]=\sum_{w=1}^{W} \sum_{\tau \in \mathcal{S}_{w}} Z[\tau] X^{\top}[\tau+\omega] \quad \text { for } \omega \in \Theta .
\end{array}
$$

For all $(\omega, \tau) \in \mathcal{V}_{\Theta}(0) \times \mathcal{S}_{w}$, we have $\omega+\tau \in \mathcal{S}_{w} \cup \mathcal{E}_{\Theta}\left(\mathcal{S}_{w}\right)$. Thus, the computations of the inner sum $\boldsymbol{\Phi}^{w}$ can be made independently by each worker. These partial results are gathered and reduced with $\boldsymbol{\Phi}=\sum_{w=1}^{W} \boldsymbol{\Phi}^{w}$. By making use of these distributed computation, $\Phi$ and $\boldsymbol{\Psi}$ can be computed with respectively $\mathcal{O}\left(K^{2}\left|\mathcal{S}_{w}\right| \log \left(\left|\mathcal{S}_{w} \cup \mathcal{E}_{\Theta}\left(\mathcal{S}_{w}\right)\right|\right)\right)$ and $\mathcal{O}\left(K P\left|\mathcal{S}_{w}\right| \log \left(\mid \mathcal{S}_{w} \cup\right.\right.$ $\left.\mathcal{E}_{\Theta}\left(\mathcal{S}_{w}\right) \mid\right)$ ) operations. The values of $\boldsymbol{\Psi}$ and $\boldsymbol{\Phi}$ are sufficient statistics to compute $\nabla_{\boldsymbol{D}} F$ and $F$. The dictionary can thus be updated without having to gather the full activation $Z$ on one machine. This allows to learn a dictionary for a signal $X$ that does not fit in memory, as each worker only needs to store a part of the full signal and the dictionary updates only require storing these sufficient statistics.

\section{Numerical EXPERIMENTS}

The numerical experiments are performed on a SLURM cluster with 30 nodes. Each node has 20 physical cores, 40 logical cores and $250 \mathrm{~GB}$ of RAM. A worker is typically one logical core on one of the machine. In practice we use up to 400 workers. DiCoDiLe is implemented in Python [26] using mpi 4py [8] ${ }^{1}$.

Experiments make use of simulated one dimensional signals, generated following the sparse convolutional linear model (2) with $d=1$ and $P=7$. The dictionary is composed of $K=25$ atoms $D_{k}$ of length $L=250$. Each atom is sampled from a standard Gaussian distribution and then normalized. The sparse code entries are drawn from a Bernoulli-Gaussian distribution, with Bernoulli parameter $\rho=0.007$, mean 0 and standard variation 10 . The noise term $\xi$ is sampled from a standard Gaussian distribution with variance 1 . The length of the signals $X$ is denoted $T$. For experiments with images, we will use the standard colored image Mandrill $(P=3)$ at full resolution $(512 \times 512)$ as well as text images generated with LATEX and ImageMagick [32]. Finally, for large scale experiments, we used an image of the Great Observatories Origins Deep Survey (GOODS) South field, acquired by the Hubble Space Telescope [13]. We used the STScI-H-2016-39 image $^{2}$ with resolution $6000 \times 3600$.

In all experiments, we used $\lambda=0.1 \lambda_{\max }$ as a default regularization parameter, unless specified otherwise.

\subsection{Performance of distributed sparse coding}

The performances of the distributed sparse coding step in DiCoDiLe is impacted by the choice of coordinate descent strategy, as well as soft-lock mechanism and the data partitioning scheme. The following experiments discuss their effects on the scalability on $\operatorname{DiCoDiLe}_{z}$.

\subsubsection{Coordinate Selection Strategy}

Using one worker, Figure 3 compares the average running times of three CD strategies to solve (4): Cyclic, Greedy and LGCD. LGCD consistently outperforms the other strategies for both $T=150 \mathrm{~L}$ and $T=750 \mathrm{~L}$. For LGCD and Cyclic, the iteration complexity is constant compared to the size of the signal, whereas the complexity of each Greedy iteration scales linearly with T. This makes running time of Greedy explode when $T$ grows. Besides, as LGCD also prioritizes the more important coordinates, it is more efficient than Cyclic that selects the coordinate to update independently of the data. This explains the performances observed in Figure 3 and justifies the use of LGCD as the base of the $\mathrm{DiCoDiLe}_{z}$ distributed algorithm. In the following, we will not include Cyclic coordinate selection in our distributed algorithm as it is less efficient. We include

1. Code available at github.com/tommoral/dicodile.

2. The image is publicly available on Hubble website: www.hubblesite.org/image/3920/news/58-hubble-ultra-deep-field 


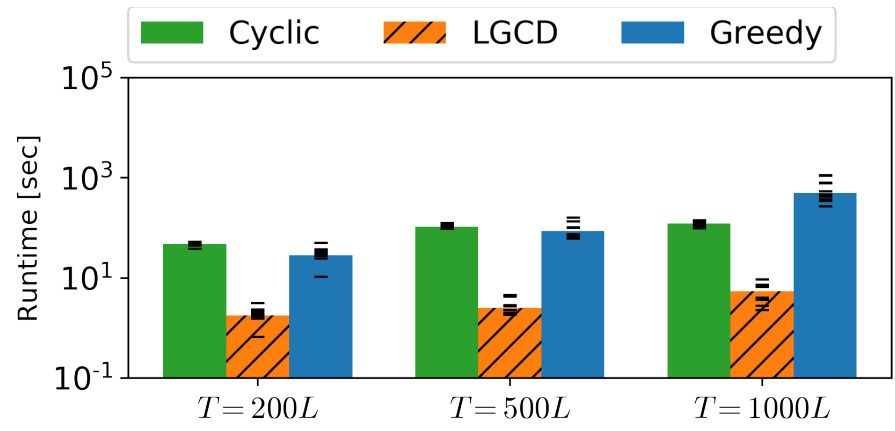

Fig. 3. Average running time of $C D$ with three coordinate selection schemes - (green) Cyclic, (orange) Locally Greedy and (blue) Greedy - for three signal lengths and $\lambda=0.5 \lambda_{\max }$. LGCD consistently outperforms the two other strategies.
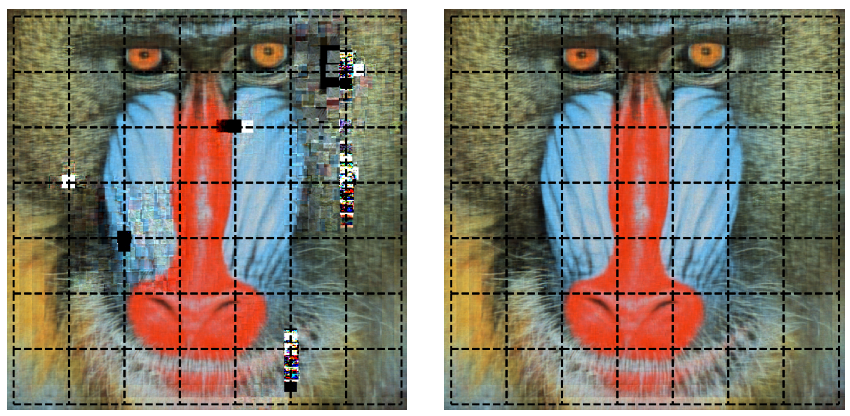

Fig. 4. Reconstruction of the image Mandrill using DiCoDiLe (left without and (right) with soft-locks for a grid of $7 \times 7$ workers. The dashed lines show the partitions of the domain $\Omega$. The algorithm diverges at the edges of some of the sub-domains due to interfering updates between more than two workers.

Greedy as its complexity depends on the size of the signal, meaning that it can be competitive when the workload is distributed on many workers.

\subsubsection{Need for soft-lock mechanism}

The reconstruction of the Mandrill with and without soft-locks is shown in Figure 4. It uses a dictionary $\boldsymbol{D}$ consisting of $K=25$ patches of size $16 \times 16$ extracted randomly from the original image. The reconstructed image $Z * \boldsymbol{D}$ obtained without soft-lock shows artifacts around the edges of some of the sub-domains $\mathcal{S}_{w}$, as the activations diverge in these locations. This visually demonstrates the need for controlling the interfering updates when more than two workers can interfere. When using the soft-lock mechanism, the algorithm returns the expected result.

\subsubsection{Impact of data partitioning}

To demonstrate the impact of the domain partitioning strategy on images, Figure 5 shows the average runtime for $\mathrm{DiCoDiLe}_{Z}$ using either a line of worker (unidirectional partitioning) or a grid of workers (bidirectional partitioning). In this experiment $K=5$ and the atom size is $8 \times 8$. Both strategies scale similarly in the regime with low number of workers. But when $W$ reaches $T_{1} / 3 L_{1}$, the performances of DiCoDiLe $Z$ stops improving when using the unidirectional partitioning of $\Omega$. Indeed in this case many update candidates are selected at the border of the sub-domains $\mathcal{S}_{w}$, therefore increasing the chance to reject an update. Moreover, the scaling of the unidirectional partitioning is limited to $W=T_{1} / 2 L_{1}=32$, whereas the bidirectional partitioning can be used with $W$ up to 1024 workers.

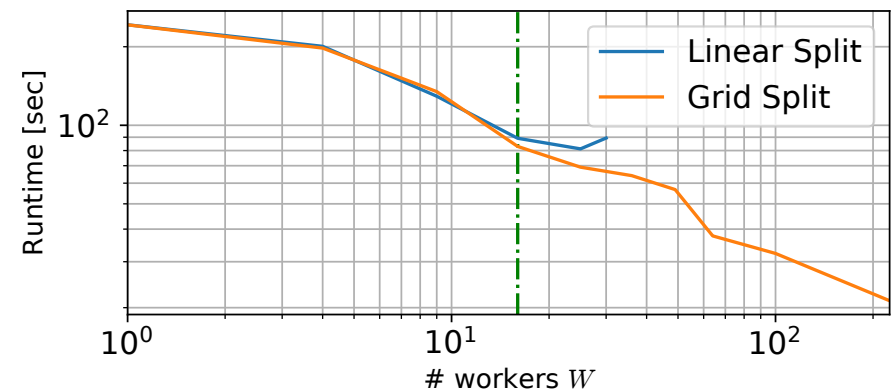

Fig. 5. Scaling on 2D images of DiCoDiLe $z$ with the number of workers for two partitioning strategies of $\Omega$ : (blue) $\Omega$ is split only along one direction, as in DICOD [21], (orange) $\Omega$ is split along both directions, on a grid. The running times are similar for low number of workers but the performances with the linear splits stop improving once $W$ reaches the scaling limit $T_{1} / 4 L_{1}$ (green). With the grid of workers adapted to images, the performance improves further. The linear split stops when $W$ reached $T_{1} / L_{1}$ because no more coordinate in $\mathcal{S}_{w}$ are independent from other neighbors.
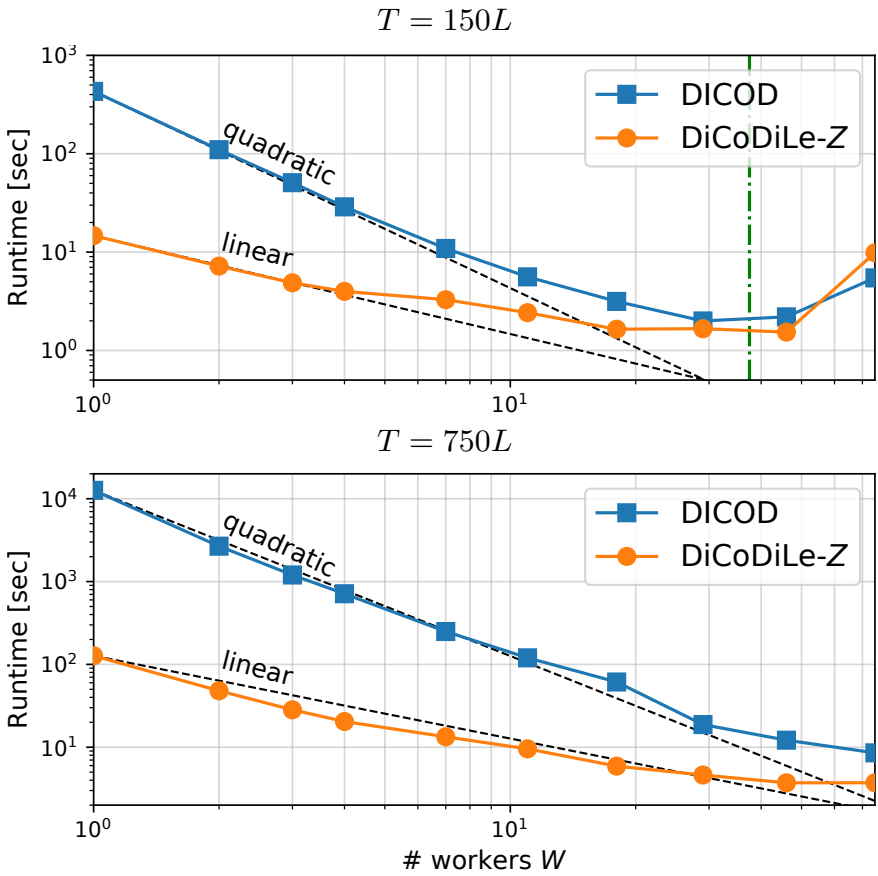

Fig. 6. Scaling of DICOD (orange) and DiCoDiLe $z$ (blue) with the number of workers $W$. DiCoDiLe $z$ scales sub-linearly while DICOD scales super-linearly. However, DiCoDiLe $z$ is more efficient than DICOD in a regime with a low number of workers $W$, leading to improved performances in all regimes. The green line denotes the number of cores where $\mathrm{DiCoDiLe}_{Z}$ and DICOD become the same as DiCoDiLe $z$ only has one sub-domain in each worker. The results are consistent for both small (top, $T=150 L$ ) and large (bottom $T=750 L$ ) signals sizes.

\subsubsection{Scaling of DiCoDiLe $e_{Z}$}

Scaling for 1D signals Figure 6 investigates the role of $\mathrm{CD}$ strategies in the distributed setting with $1 \mathrm{D}$ signals. The average runtime of DICOD (with GCD on each worker), and $\operatorname{DiCoDiLe}_{Z}$ (with LGCD) are reported in Figure 6. DiCoDiLe $Z$ with LGCD scales sub-linearly with the number of workers used, whereas DICOD scales almost quadratically. However, $\operatorname{DiCoDiLe}_{z}$ outperforms DICOD consistently, as the performance of GCD is poor for low number of workers using large sub-domains. The two coordinate selection strategies become equivalent when $W$ reaches $T / 4 L$, as in this case DiCoDiLe 


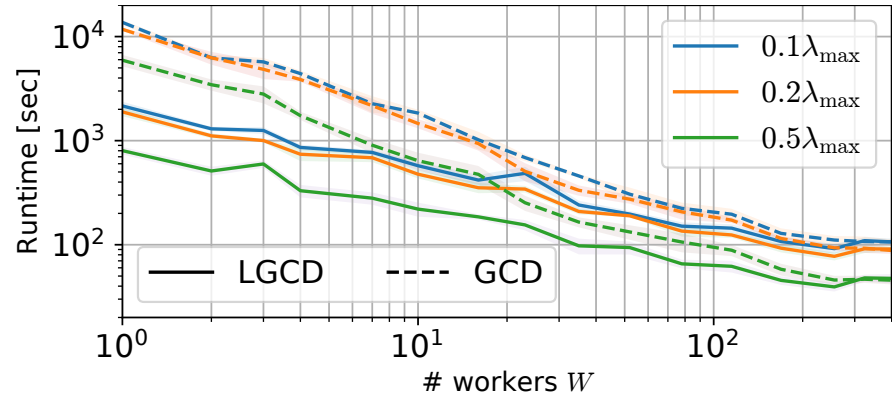

Fig. 7. Scaling of DiCoDiLe ${ }_{Z}$ with the number of workers $W$ for different values of $\lambda$ and for two strategies of coordinate selection: Greedy and Locally Greedy. The convergence is faster when $\lambda$ is large because the activation becomes sparser and less coordinates need to be updated. Also, the locally greedy coordinate selection outperforms the greedy selection up to a certain point where the performances become similar as the sub-domain $\mathcal{S}_{w}$ becomes too small to be further partitioned with sub-domains of size $\Theta$.

has only one sub-domain $\mathcal{C}_{1}^{(w)}$. This is consistent for larger signals. Indeed, in the case where $T=750, \mathrm{DiCoDiLe}_{z}$ scales linearly with the number of workers. We do not see the drop in performance, as in this experiments $W$ does not reach the scaling limit $T / 4 L$. Importantly, DiCoDiLe $z$ still outperforms DICOD for all regimes.

Scaling for 2D signals Figure 7 illustrates the scaling performance of $\mathrm{DiCoDiLe}_{Z}$ on images. The average running times of $\operatorname{DiCoDiLe}_{z}$ are reported as a function of the number of workers $W$ for different regularization parameters $\lambda$ and for greedy and locally greedy selection. As for the 1D CSC problem, the locally greedy selection consistently outperforms the greedy selection. This is particularly the case when using low number of workers $W$. As for signals, once the size of the sub-domains becomes smaller, the performances of both coordinate selection methods become closer as both algorithms become equivalent. One can also notice that the convergence of $\mathrm{DiCoDiLe}_{z}$ is faster for large $\lambda$. This is expected, as in this case, the solution $Z^{*}$ is sparser and less coordinates need to be updated.

\subsection{Comparison with Skau and Wohlberg [28]}

Figure 8 displays the evolution of the objective function (3) as a function of time for DiCoDiLe and the Consensus ADMM algorithm $^{3}$ proposed by Skau and Wohlberg [28]. To the best of our knowledge, the work of Skau and Wohlberg [28] is the only approach in the literature that proposes a parallel algorithm for CDL. Both algorithms were run on a single node with 36 workers. We used the Hubble Space Telescope GOODS South image as input data. As the algorithm by Skau and Wohlberg [28] raised a memory error with the full image on a machine with $250 \mathrm{~GB}$ of RAM, we used a random-patch of size $512 \times 512$ as input data. Due to the non-convexity of the problem, the algorithms converge to different local minima, even when initialized with the same dictionary. To deal with this difficulty, the Figure 8 shows 5 restarts of both algorithms, as well as the median curve, obtained by interpolation. We can see that DiCoDiLe outperforms the Consensus ADMM as it converges faster and to a better local minima. To ensure a fair comparison, the objective function is computed after projecting back the atoms of the dictionary $\boldsymbol{D}_{k}$ to the $\ell_{2}$-ball by scaling it with $\left\|\boldsymbol{D}_{k}\right\|_{2}^{2}$. This implies that we also needed to scale $Z$ by

3. Code available at https://sporco.readthedocs.io/

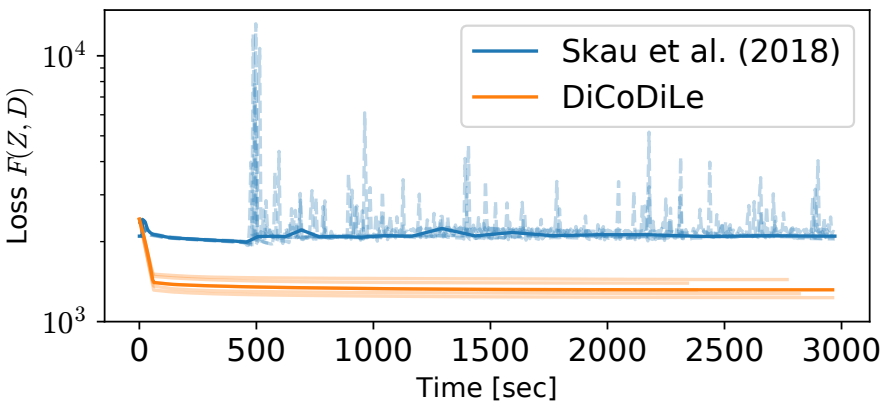

Fig. 8. Comparison of DiCoDiLe algorithm with Consensus ADMM dictionary learning proposed by Skau and Wohlberg [28]. Algorithms were run 5 times with 36 workers on a random-patch of size $512 \times 512$ taken from the Hubble Space Telescope GOODS South image. The solid lines denote the median curves obtained through interpolation.

the inverse of the dictionary norm i.e. $\frac{1}{\left\|\boldsymbol{D}_{k}\right\|_{2}^{2}}$. This projection and scaling step is necessary for the solver as ADMM iterations do not guarantee that all iterates are feasible (i.e., satisfy the constraints). This also explains the presence of several bumps in the evolution curve of the objective function.

\subsection{Pattern recovery with large images}

To illustrate the benefit of working with large images, we propose two pattern recovery experiments on text images and on an image of the Hubble Space Telescope.

Text images Figure 9 presents the patterns that are recovered using CDL and Online Dictionary Learning (Online $\mathrm{DL}^{4}$, Mairal et al. 20) from a text image composed of the letters PAMI. The text is generated by drawing uniformly 5000 characters from a set composed of these $K^{*}=4$ letters and 2 spaces. Text images $X$, mimicking documents obtained with a typewritter, are generated from this text using $\mathrm{HT}_{\mathrm{E}} \mathrm{X}$ and font beramono. They are then converted to PNG using ImageMagick [32]. We add a Gaussian white noise with standard deviation $\sigma \operatorname{std}(X)$ and $\sigma=3$ to $X$ (see Figure 9 bottom). The figure presents the qualitative results obtained by drawing uniformly $K=10$ patches (dashed green), or learning $K$ patterns with DiCoDiLe (orange; $\lambda=0.2$ ) and Online DL (blue; $\lambda=0.1$ ). The quality of the recovered atoms is evaluated using $\rho$, the convolutional cosine similarity following optimal assignment defined as

$$
\min _{\sigma \in \mathfrak{S}_{K}} \sum_{l=1}^{K^{*}} \rho\left(d_{l}^{*}, d_{\sigma(l)}\right) ; \quad \rho(d, \hat{d})=\max _{t \in \mathcal{V}_{\Theta}(0)} \sum_{\tau \in \Theta}^{W} d[t+\tau] \hat{d}[\tau] .
$$

The atoms that are presented for each line are selected with the argmin permutation in (25). This argmin over the set of permutations is computed using the so-called Hungarian algorithm [19]. While DiCoDiLe is able to successfully recover the original patterns, Online DL learns blurred atoms that are unrecognizable. This highlights one of the main interest of CDL over ordinary DL, which is its ability to capture the patterns in the data in a shift invariant way. Indeed, as DL encodes all patches in a image, the recovered patterns are inevitably blurred as all phases of a pattern are of equal importance. In contrast, CDL selects specific shifts that are used to compute the atoms, allowing for sharper pattern recovery.

To assess the impact of the image size on the performance of CDL with DiCoDiLe, we generated images following the same protocol as before but drawing uniformly $K^{*}=5$ letters from

4. Code available at http://spams-devel.gforge.inria.fr 

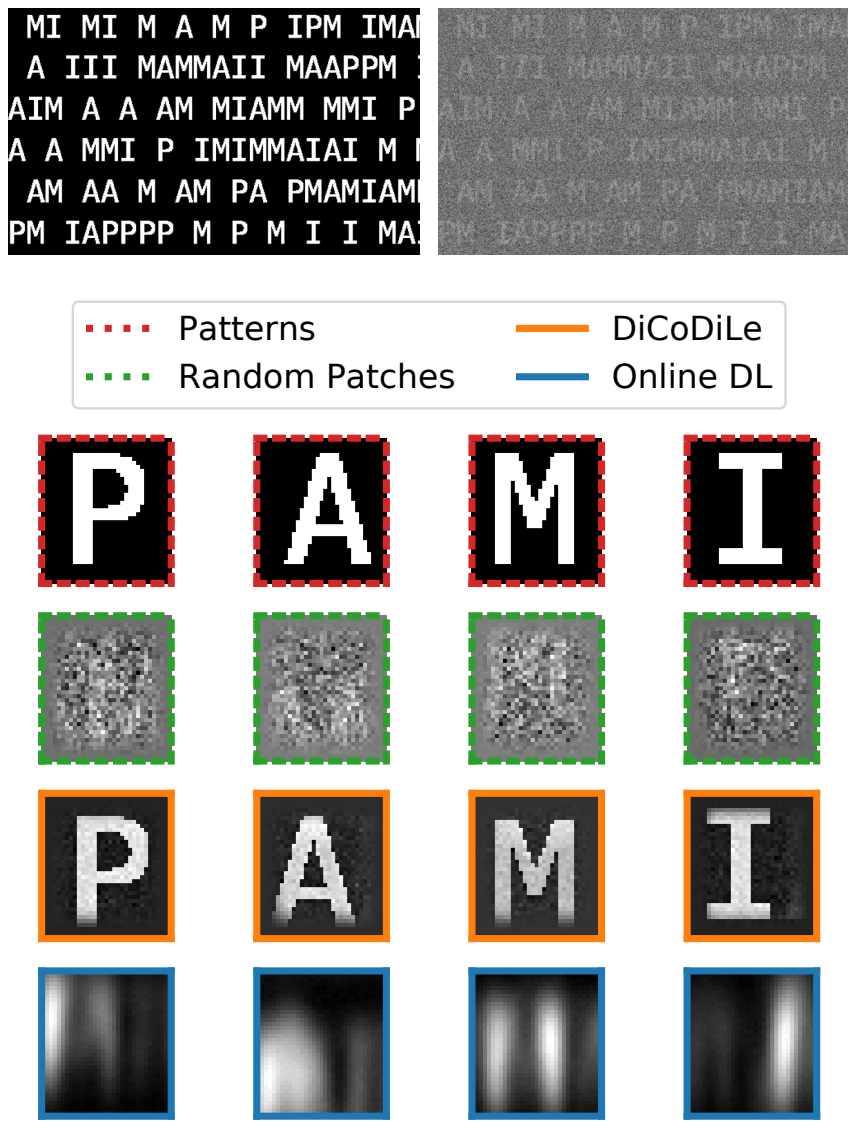

Fig. 9. Letter recovery experiment using a noisy image of a text with 5000 characters drawn uniformly from PAMI (top). For each row, the atoms that are displayed have been selected among $K=10$ with the best assignment $\rho$ with the original patterns (dashed red). Random patches drawn uniformly from the original image (dashed green) are used as initial estimates $D_{0}$. We use DiCoDiLe (orange) and Online DL (blue, [20]) to learn $K=10$ atoms. While the atoms recovered with Online DL are blurred, CDL is able to correcly recover the 4 original letters.

$a-z$ and then generating text with these letters for varying text-length before converting them to images $X$. A Gaussian white noise with standard deviation sigma $* \operatorname{std}(X)$ is then added to the image. Figure 10 presents for varying text sizes the recovery score $\rho$ averaged on 10 repetition for Online DL (blue) and DiCoDiLe (orange) both with $\lambda=0.2$. We also report the performances obtained with random patches (dashed green) extracted from the noisy input image $X$ and with random normal patches (dashed black) obtained by sampling each pixel from a normal distribution $\mathcal{N}(.5,1)$. For low level of noise $\sigma=.1$ (left), the recovery of the patterns with CDL is already very close to 1 with only small images (0.2 Mpx) with 150 characters. We see here that CDL consistently outperforms DL which have a performance a bit better than extracting random patches from the image. For large level of noise $\sigma=3$ (right), we see that the random patches are very noisy and perform worst than normal patches that are centered around 0.5. Both Online DL and CDL improves with the size of the image as they take advantage of the repetition of the patterns to separate them from the noise. DiCoDiLe is able to consistently recover the shape of the original patterns, up to a level close to the one obtained in noiseless setting for very large images (4.8 Mpx) with 5000 characters.

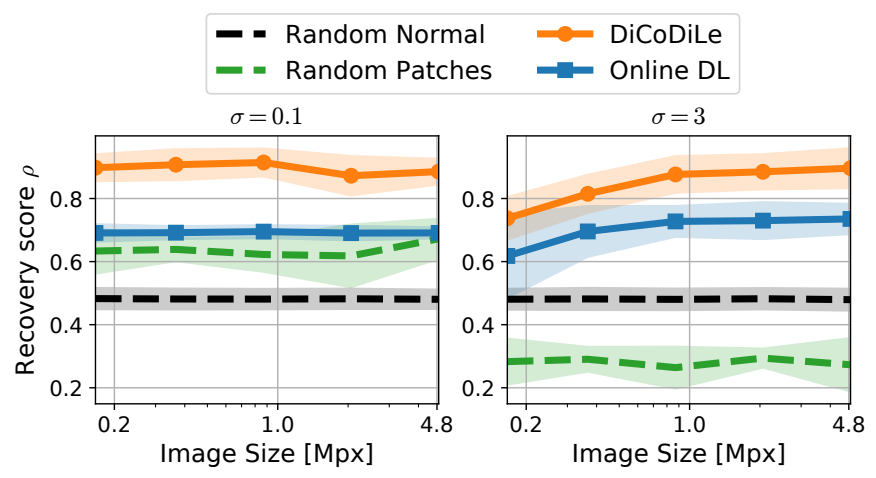

Fig. 10. Effect of the image size (in Megapixels) on letter recovery for two noise levels. The quality of the recovered patterns is evaluated with best assignment $\rho$. One observes that increasing image size improves recovery of the patterns which are here letters, as the number of characters present in the image increases.

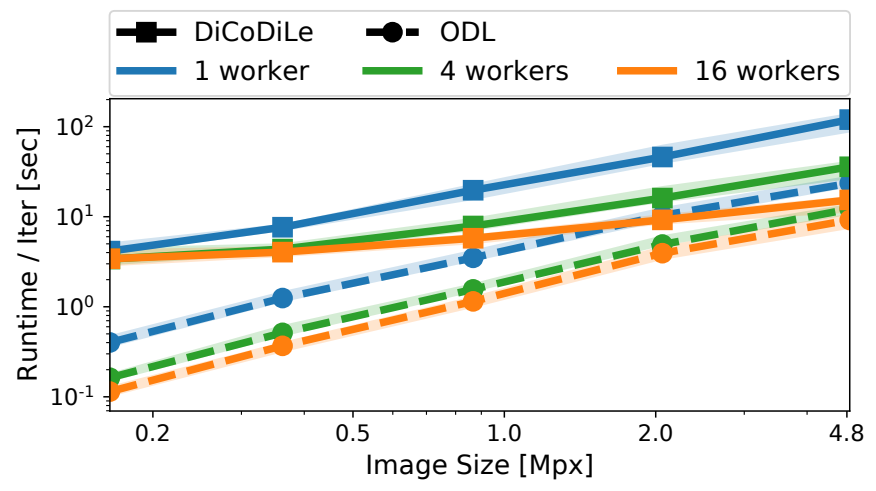

Fig. 11. Median running time of one iteration of DiCoDiLe $(Z$ and $D$ steps) and one epoch of Online DL for text images with different number of workers $W$ and image sizes (in Megapixels). While the running times for DiCoDiLe with any number of workers are all similar for small images, there is a significant speed up when using multiple workers for larger images.

This experiment clearly demonstrates the need for CDL algorithms that are able to scale to large input data, as it is a necessary condition to design efficient pattern recovery system. We then report running times of DiCoDiLe and Online DL for this use case.

Figure 11 displays the running time per iteration for DiCoDiLe and per epoch for Online DL with $W$ in $\{1,4,16\}$ for the text images used in the previous experiment. We consider data with $\sigma=3$ and different image sizes. The reported running times are the median time over 10 repetitions of the experiment. In this figure, we can see that for small images, the running time of one iteration of DiCoDiLe is similar for any number of workers. This can be explained by the fact that the cost of the dictionary update step is dominating the computations. Also using a lot of workers for small images increases the number of interfering updates - thus reducing the benefit of parallelism. However, when the size of the images increases, the cost of the $Z$ step increases and using DiCoDiLe with more than $W=1$ worker reduces massively the running time - up to 10 times for larger images with 16 workers. In comparison, the benefit of using more workers is constant for Online DL. For large images where one epoch amounts to many patches, the running time of Online DL with 16 workers for one epoch becomes similar to the one of DiCoDiLe with the same number of workers. This 


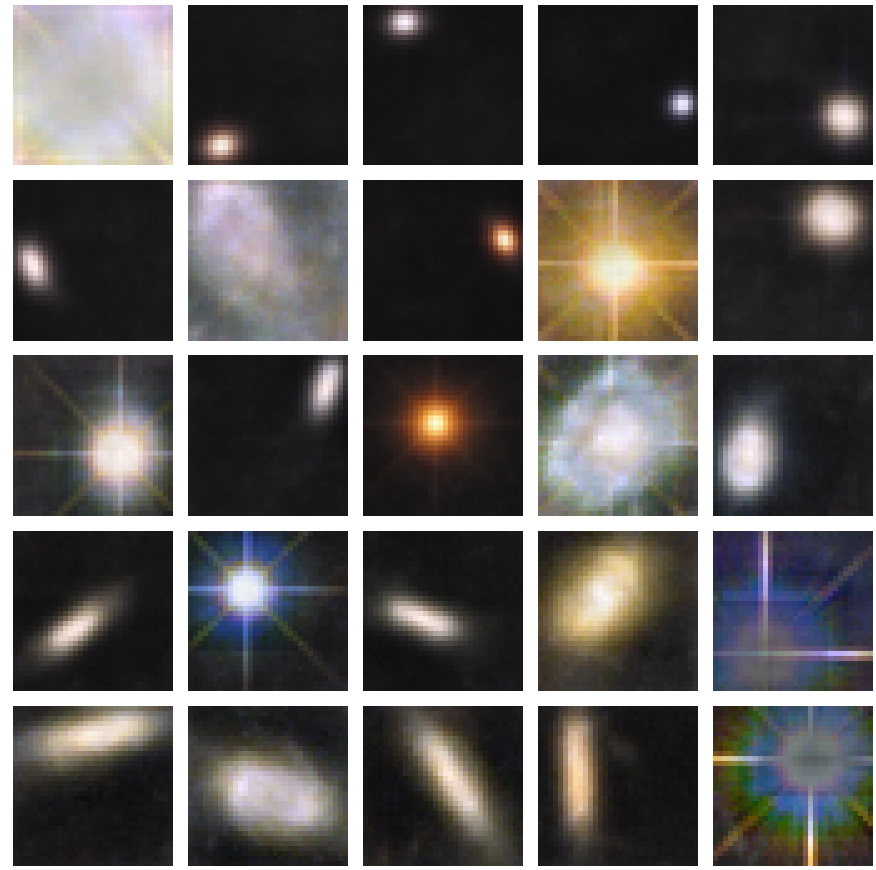

Fig. 12. 25 atoms $32 \times 32$ learned from the Hubble Space Telescope images (STSCI-H-2016-39-a) using DiCoDiLe with 400 workers and a regularization parameter $\lambda=0.1 \lambda_{\max }$. The atoms are sorted based on $\left\|Z_{k}\right\|_{1}$ from the top-left to the bottom-right. Most atoms displays a structure which corresponds to spatial objects. The atoms 1 and 7 have fuzzier structure because they are mainly used to encode larger scale objects.

experiment demonstrates that DiCoDiLe is able to significantly accelerate the resolution of CDL for large images by leveraging distributed computations, and can be competitive with Online DL in term of computational performances.

Hubble Telescope images We present here results using DiCoDiLe to learn patterns from an image of the GOODS South field, acquired by the Hubble Space Telescope [13]. We used $W=400$ workers to learn 25 atoms of size $32 \times 32$ with regularization parameter $\lambda=0.1 \lambda_{\max }$. The learned patterns are presented in Figure 12. This unsupervised algorithm is able to highlight structured patterns in the image, such as small stars. Patterns 1 and 7 are very fuzzy. This is probably due to the presence of very large object in the foreground. As this algorithm is not scale invariant, the objects larger than our patterns are encoded using these low frequency atoms.

\section{Conclusion}

This work introduces DiCoDiLe, an asynchronous distributed algorithm to apply convolutional dictionary learning to very large multidimensional data such as signals and images. The number of workers that can be used to solve the CSC scales linearly with the data size, allowing to adapt the computational resources to the problem dimensions. Using careful distributed pre-computations, the complexity of the dictionary update step is independent of the data size. Experiments show that the proposed algorithm outperforms the current state-of-theart parallel algorithms for CDL. Thanks to its good scaling properties, DiCoDiLe is able to process large data such as astronomical images, improving the unsupervised recovery of recurrent patterns in large datasets. Finally, recent development are pushing to refine CDL models such as the multi-layer CSC [30, 31], CSC with strided convolution [27] or rank-1
CDL [10]. These models rely on a similar encoding step that can be computed efficiently with our distributed method, opening for further investigations and improved implementations.

\section{APPENDIX A \\ COMPUTATION FOR THE COST UPDATES}

When a coordinate $Z_{k}[\omega]$ is updated to $\widetilde{Z}_{k}^{(q)}[\omega]$, the cost update is a simple function of $Z_{k}[\omega]$ and $\widetilde{Z}_{k}^{(q)}[\omega]$.

Proposition A.1. If the coordinate $\left(k_{0}, \omega_{0}\right)$ is updated from value $Z_{k_{0}}\left[\omega_{0}\right]$ to value $\widetilde{Z}_{k_{0}}^{(q)}\left[\omega_{0}\right]$, then the change in the cost function $\Delta E_{k_{0}}\left[\omega_{0}\right]$ is given by:

$$
\begin{aligned}
\Delta E_{k_{0}}\left[\omega_{0}\right]= & \frac{\left\|\boldsymbol{D}_{k_{0}}\right\|_{2}^{2}}{2}\left(Z_{k_{0}}\left[\omega_{0}\right]^{2}-\widetilde{Z}_{k_{0}}^{(q)}\left[\omega_{0}\right]^{2}\right) \\
& -\beta_{k_{0}}\left[\omega_{0}\right]\left(Z_{k_{0}}\left[\omega_{0}\right]-\widetilde{Z}_{k_{0}}^{(q)}\left[\omega_{0}\right]\right) \\
& +\lambda\left(\left|Z_{k_{0}}\left[\omega_{0}\right]\right|-\left|\widetilde{Z}_{k_{0}}^{(q)}\left[\omega_{0}\right]\right|\right) .
\end{aligned}
$$

Proof. We denote $Z_{k}^{(1)}[\omega]= \begin{cases}\widetilde{Z}_{k_{0}}^{(q)}\left[\omega_{0}\right], & \text { if }(k, \omega)=\left(k_{0}, \omega_{0}\right) \\ Z_{k}[\omega], & \text { elsewhere }\end{cases}$ Let $\alpha_{0}$ be the residual when coordinate $\left(k_{0}, \omega_{0}\right)$ of $Z$ is set to 0 . For $\omega \in \Omega$,

$$
\begin{aligned}
\alpha_{k_{0}}[\omega] & =(X-Z * \boldsymbol{D})[\omega]+\boldsymbol{D}_{k_{0}}\left[\omega-\omega_{0}\right] Z_{k_{0}}\left[\omega_{0}\right] \\
& =(X-Z * \boldsymbol{D})[\omega]+Z_{k_{0}}\left[\omega_{0}\right]\left(e_{\omega_{0}} * \boldsymbol{D}_{k_{0}}\right)\left[\omega_{0}\right]
\end{aligned}
$$

We also have $\alpha_{k_{0}}[\omega]=\left(X-Z^{(1)} * \boldsymbol{D}\right)[\omega]+\boldsymbol{D}_{k_{0}}\left[\omega-\omega_{0}\right] \widetilde{Z}_{k_{0}}^{(q)}\left[\omega_{0}\right]$. Then

$$
\begin{aligned}
\Delta E_{k_{0}}\left[\omega_{0}\right]= & \frac{1}{2} \sum_{\omega \in \Omega}(X-Z * \boldsymbol{D})^{2}[\omega]+\lambda\|Z\|_{1} \\
& -\frac{1}{2} \sum_{\omega \in \Omega}\left(X-Z^{(1)} * \boldsymbol{D}\right)^{2}[\omega]+\lambda\left\|Z^{(1)}\right\|_{1} \\
= & \frac{1}{2} \sum_{\omega \in \Omega}\left(\alpha_{k_{0}}[\omega]-\boldsymbol{D}_{k_{0}}\left[\omega-\omega_{0}\right] Z_{k_{0}}\left[\omega_{0}\right]\right)^{2} \\
& -\frac{1}{2} \sum_{\omega \in \Omega}\left(\alpha_{k_{0}}[\omega]-\boldsymbol{D}_{k_{0}}\left[\omega-\omega_{0}\right] \widetilde{Z}_{k_{0}}^{(q)}\left[\omega_{0}\right]\right)^{2} \\
& +\lambda\left(\left|Z_{k_{0}}\left[\omega_{0}\right]\right|-\left|\widetilde{Z}_{k_{0}}^{(q)}\left[\omega_{0}\right]\right|\right) \\
= & \frac{1}{2} \sum_{\omega \in \Omega} \boldsymbol{D}_{k_{0}}\left[\omega-\omega_{0}\right]^{2}\left(Z_{k_{0}}\left[\omega_{0}\right]^{2}-\widetilde{Z}_{k_{0}}^{(q)}\left[\omega_{0}\right]^{2}\right) \\
& -\sum_{\omega \in \Omega} \alpha_{k_{0}}[\omega] \boldsymbol{D}_{k_{0}}\left[\omega-\omega_{0}\right]\left(Z_{k_{0}}\left[\omega_{0}\right]-\widetilde{Z}_{k_{0}}^{(q)}\left[\omega_{0}\right]\right) \\
& +\lambda\left(\left|Z_{k_{0}}\left[\omega_{0}\right]\right|-\left|\widetilde{Z}_{k_{0}}^{(q)}\left[\omega_{0}\right]\right|\right) \\
= & \frac{\left\|\boldsymbol{D}_{k_{0}}\right\|_{2}^{2}}{2}\left(Z_{k_{0}}\left[\omega_{0}\right]^{2}-\widetilde{Z}_{k_{0}}^{(q)}\left[\omega_{0}\right]^{2}\right) \\
& -\underbrace{\left(\alpha k_{k_{0}} * \boldsymbol{D}_{k_{0}}^{\dagger}\right)\left[\omega_{0}\right]}_{\beta_{k_{0}}\left[\omega_{0}\right]}\left(Z_{k_{0}}\left[\omega_{0}\right]-\widetilde{Z}_{k_{0}}^{(q)}\left[\omega_{0}\right]\right) \\
& +\lambda\left(\left|Z_{k_{0}}\left[\omega_{0}\right]\right|-\left|\widetilde{Z}_{k_{0}}^{(q)}\left[\omega_{0}\right]\right|\right) \\
&
\end{aligned}
$$

This concludes our proof. 
Using this result, we can derive the optimal value $\widetilde{Z}_{k_{0}}^{(q)}\left[\omega_{0}\right]$ to update the coordinate $\left(k_{0}, \omega_{0}\right)$ as the solution of the following optimization problem:

$$
\begin{aligned}
\widetilde{Z}_{k_{0}}^{(q)}\left[\omega_{0}\right] & =\arg \min _{y \in \mathbb{R}} e_{k_{0}, \omega_{0}}(u) \\
& =\arg \min _{u \in \mathbb{R}} \frac{\left\|\boldsymbol{D}_{k_{0}}\right\|_{2}^{2}}{2}\left(u-\beta_{k_{0}}\left[\omega_{0}\right]\right)^{2}+\lambda|u| .
\end{aligned}
$$

In the case where multiple coordinates $\left(k_{w}, \omega_{w}\right)$ are updated in the same iteration to values $\widetilde{Z}_{k_{w}}^{(q)}\left[\omega_{w}\right]$, we obtain the following cost variation.

Proposition A.2. The update of the $W$ coordinates $\left(k_{w}, \omega_{w}\right)_{w=1}^{W}$ with additive update $\Delta Z_{k_{w}}\left[\omega_{w}\right]$ changes the cost by:

$$
\begin{aligned}
\Delta E= & \overbrace{\sum_{i=1}^{W} \Delta E_{w}}^{\text {iterative steps }} \\
& -\underbrace{\sum_{w \neq w^{\prime}}\left(\boldsymbol{D}_{k_{w}} * \boldsymbol{D}_{k_{w^{\prime}}}^{\dagger}\right)\left[\omega_{w^{\prime}}-\omega_{w}\right] \Delta Z_{k_{w}}\left[\omega_{w}\right] \Delta Z_{k_{w^{\prime}}}\left[\omega_{w^{\prime}}\right]}_{\text {interference }},
\end{aligned}
$$

Proof. We define $Z_{k}^{(1)}[\omega]=\left\{\begin{array}{ll}\widetilde{Z}_{k_{w}}^{(q)}\left[\omega_{w}\right], & \text { if }(k, \omega)=\left(k_{w}, \omega_{w}\right) \\ Z_{k}[\omega], & \text { otherwise }\end{array}\right.$. Let

$$
\begin{aligned}
\gamma[\omega] & =(X-Z * \boldsymbol{D})[\omega]+\sum_{w=1}^{K} \boldsymbol{D}_{k_{w}}\left[\omega-\omega_{w}\right] Z_{k_{w}}\left[\omega_{w}\right] \\
& =\left(X-Z^{(1)} * \boldsymbol{D}\right)[\omega]+\sum_{w=1}^{K} \boldsymbol{D}_{k_{w}}\left[\omega-\omega_{w}\right] \widetilde{Z}_{k_{w}}^{(q)}\left[\omega_{w}\right],
\end{aligned}
$$

and

$$
\begin{aligned}
\alpha_{w}[\omega] & =(X-Z * \boldsymbol{D})[\omega]+\boldsymbol{D}_{k_{w}}\left[\omega-\omega_{w}\right] Z_{k_{w}}\left[\omega_{w}\right] \\
& =\gamma[\omega]-\sum_{w^{\prime} \neq w} \boldsymbol{D}_{k_{w^{\prime}}}\left[\omega-\omega_{w^{\prime}}\right] Z_{k_{w^{\prime}}}\left[\omega_{w^{\prime}}\right] .
\end{aligned}
$$

Then

$$
\begin{aligned}
\Delta E= & \frac{1}{2} \sum_{\omega \in \Omega}(X[\omega]-Z * \boldsymbol{D}[\omega])^{2}+\lambda\|Z\|_{1} \\
& -\sum_{\omega \in \Omega}\left(X[\omega]-Z^{(1)} * \boldsymbol{D}[\omega]\right)^{2}-\lambda\left\|Z^{(1)}\right\|_{1} \\
= & \frac{1}{2} \sum_{\omega \in \Omega}\left(\gamma[\omega]-\sum_{w=1}^{W} \boldsymbol{D}_{k_{w}}\left[\omega-\omega_{w}\right] Z_{k_{w}}\left[\omega_{w}\right]\right)^{2} \\
& -\frac{1}{2} \sum_{\omega \in \Omega}\left(\gamma[\omega]-\sum_{w=1}^{W} \boldsymbol{D}_{k_{w}}\left[\omega-\omega_{w}\right] \widetilde{Z}_{k_{w}}^{(q)}\left[\omega_{w}\right]\right)^{2} \\
& +\lambda \sum_{w=1}^{W}\left(\left|Z_{k_{w}}\left[\omega_{w}\right]\right|-\left|\widetilde{Z}_{k_{w}}^{(q)}\left[\omega_{w}\right]\right|\right) \\
= & \frac{1}{2} \sum_{w=1}^{W}\left(\sum_{\omega \in \Omega} \boldsymbol{D}_{k_{w}}\left[\omega-\omega_{w}\right]^{2}\right)\left(Z_{k_{w}}\left[\omega_{w}\right]^{2}-\widetilde{Z}_{k_{w}}^{(q)}\left[\omega_{w}\right]^{2}\right) \\
& +\lambda \sum_{w=1}^{W}\left(\left|Z_{k_{w}}\left[\omega_{w}\right]\right|-\left|\widetilde{Z}_{k_{w}}^{(q)}\left[\omega_{w}\right]\right|\right) \\
& -\sum_{w=1}^{W} \sum_{\omega \in \Omega}\left(\gamma[\omega] \boldsymbol{D}_{k_{w}}\left[\omega-\omega_{w}\right]\right) \Delta Z_{k_{w}}\left[\omega_{w}\right] \\
& -\sum_{w \neq w^{\prime}}\left[\sum_{\omega \in \Omega}\left(\boldsymbol{D}_{k_{w}}\left[\omega-\omega_{w}\right] \boldsymbol{D}_{k_{w}}\left[\omega-\omega_{w^{\prime}}\right]\right)\right. \\
& \\
&
\end{aligned}
$$

$$
\begin{aligned}
& \left.\times\left(Z_{k_{w}}\left[\omega_{w}\right] Z_{k_{w^{\prime}}}\left[\omega_{w^{\prime}}\right]-\widetilde{Z}_{k_{w}}^{(q)}\left[\omega_{w}\right] \widetilde{Z}_{k_{w^{\prime}}}^{(q)}\left[\omega_{w^{\prime}}\right]\right)\right] \\
& =\sum_{w=1}^{W}\left[\frac{\left\|\boldsymbol{D}_{k_{w}}\right\|_{2}^{2}}{2}\left(Z_{k_{w}}\left[\omega_{w}\right]^{2}-\widetilde{Z}_{k_{w}}^{(q)}\left[\omega_{w}\right]^{2}\right)\right. \\
& -(\underbrace{\sum_{\omega \in \Omega} \alpha_{k_{w}}[\omega] \boldsymbol{D}_{k_{w}}\left[\omega-\omega_{w}\right]}_{\alpha_{k_{w}} * \boldsymbol{D}_{k_{w}}^{\uparrow}\left[\omega_{w}\right]}) \Delta Z_{k_{w}}\left[\omega_{w}\right] \\
& \left.+\lambda\left(\left|Z_{k_{w}}\left[\omega_{w}\right]\right|-\left|\widetilde{Z}_{k_{w}}^{(q)}\left[\omega_{w}\right]\right|\right)\right] \\
& -\sum_{w \neq w^{\prime}}\left[\left(\boldsymbol{D}_{k_{w^{\prime}}} * \boldsymbol{D}_{k_{w}}^{\dagger}\right)\left[\omega_{w}-\omega_{w^{\prime}}\right]\right. \\
& \times\left(\Delta Z_{k_{w}}\left[\omega_{w}\right] \widetilde{Z}_{k_{w^{\prime}}}^{(q)}\left[\omega_{w^{\prime}}\right]+\Delta Z_{k_{w^{\prime}}}\left[\omega_{w^{\prime}}\right] \widetilde{Z}_{k_{w}}^{(q)}\left[\omega_{w}\right]\right. \\
& \left.\left.-Z_{k_{w}}\left[\omega_{w}\right] Z_{k_{w^{\prime}}}\left[\omega_{w^{\prime}}\right]-\widetilde{Z}_{k_{w}}^{(q)}\left[\omega_{w}\right] \widetilde{Z}_{k_{w^{\prime}}}^{(q)}\left[\omega_{w^{\prime}}\right]\right)\right] \\
& =\sum_{w=1}^{W} \Delta E_{k_{w}}\left[\omega_{w}\right] \\
& -\sum_{w \neq w^{\prime}}\left(\boldsymbol{D}_{k_{w^{\prime}}} * \boldsymbol{D}_{k_{w}}^{\dagger}\right)\left[\omega_{w}-\omega_{w^{\prime}}\right] \Delta Z_{k_{w}}\left[\omega_{w}\right] \Delta Z_{k_{w^{\prime}}}\left[\omega_{w^{\prime}}\right]
\end{aligned}
$$

\section{APPENDIX B}

\section{Probability of a coordinate to BE SOFT-LOCKED}

Proposition B.1. We consider DiCoDiLe $z$ with $W$ workers $\left(W_{i}\right.$ in each direction), run on a signal with d dimensions. We denote $T_{i}$ the length of the signal and $L_{i}$ the length of the atoms in each dimension $i$. Assuming that the updates are spread uniformly on $\Omega$, the probability that an update candidate at random localization $\omega$ is accepted is lower bounded by

$$
P(\omega \notin S L) \geq \prod_{i=1}^{d}\left(1-\frac{W_{i} L_{i}}{T_{i}}\right)
$$

Proof. We denote $\omega_{0}$ the position of an update candidate, and $\mathcal{W}_{\omega_{0}}=\left\{w \in[1, W]\right.$ s.t. $\left.\mathcal{S}_{w} \cap \mathcal{V}_{\Theta}(\omega)=\emptyset\right\}$ the set of workers that are impacted by this update. We denote $j_{\omega_{0}}=\left|\mathcal{W}_{\omega_{0}}\right|$ the number of workers in this set. With the definition of the softlock, there exists at least one worker $w$ in $\mathcal{W}_{\omega_{0}}$ which has a coordinate that is not soft-locked. As the updates are uniformly spread across the workers, the probability of an update in $\omega_{0}$ being accepted satisfies $P\left(\omega \notin \mathbf{S L} \mid \omega=\omega_{0}\right) \geq \frac{1}{j_{\omega_{0}}}$.

Since one assumes uniform update distribution, the probability to update a coordinate at location $\omega_{0}$ is $P\left(\omega_{0}\right)=\frac{1}{|\Omega|}$. The probability for an update candidate at a random location $\omega$ to be accepted satisfies

$$
\begin{aligned}
P(\omega \notin \mathbf{S L}) & =\sum_{w=1}^{W} \sum_{\omega_{0} \in \mathcal{S}_{w}} P\left(\omega \notin \mathbf{S L} \mid \omega=\omega_{0}\right) P\left(\omega_{0}\right) \\
& \geq \sum_{w=1}^{W} \sum_{\omega_{0} \in \mathcal{S}_{w}} \frac{1}{j_{\omega_{0}}} \frac{1}{|\Omega|} \\
& \geq \frac{1}{|\Omega|} \sum_{w=1}^{W} \prod_{i=1}^{d}\left(\frac{T_{i}}{W_{i}}-L_{i}\right) \\
& \geq \frac{1}{|\Omega|} \prod_{i=1}^{d}\left(T_{i}-W_{i} L_{i}\right) \\
& \geq \prod_{i=1}^{d}\left(1-\frac{W_{i} L_{i}}{T_{i}}\right)
\end{aligned}
$$


where (B.3) results from the summation on $\Omega$. Basically, in each worker $w$, there is $\prod_{i=1}^{d}\left(T_{i} / W_{i}-2 L_{i}\right)$ coordinates for which $j_{\omega_{0}}=1$. This corresponds to the coordinate in the worker that are not on the border i.e. $\mathcal{S}_{w} \backslash \mathcal{B}_{\Theta}\left(\mathcal{S}_{w}\right)$. For a coordinate at the border with $j_{\omega_{0}}=k>1$, it suffices to notice that it will appear $\mathrm{k}$ times in the sum due to symmetry considerations. This is illustrated in Figure 13.

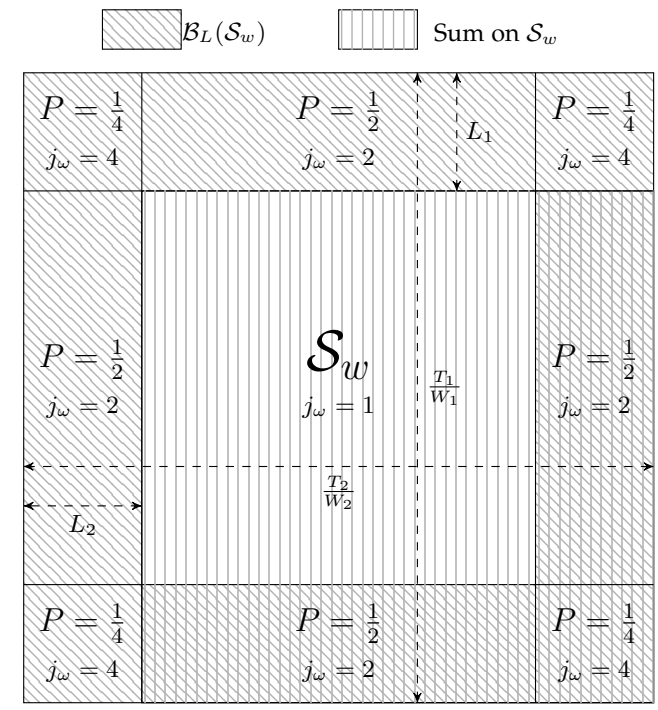

Fig. 13. Summary of the value of $j_{\omega}$ on a 2D example. We can see that the sum along each direction is $\frac{T_{i}}{W_{i}}-L_{i}$ on each workers. When multiplied by the number of workers $W_{i}$ along each direction, we obtain (B.4).

\section{ACKNOWLEDGMENTS}

This work was supported by the ERC Starting Grant SLAB ERC-StG-676943.

\section{REFERENCES}

[1] M. Aharon, M. Elad, and A. M. Bruckstein, "K-SVD: An Algorithm for Designing Overcomplete Dictionaries for Sparse Representation," IEEE Transaction on Signal Processing, vol. 54, no. 11, pp. 4311-4322, 2006.

[2] A. Beck and M. Teboulle, "A Fast Iterative ShrinkageThresholding Algorithm for Linear Inverse Problems," SIAM Journal on Imaging Sciences, vol. 2, no. 1, pp. 183-202, 2009.

[3] J. Bergstra, A. Courville, and Y. Bengio, "The Statistical Inefficiency of Sparse Coding for Images (or One Gabor to Rule them All)," preprint ArXiv, vol. 1109.6638, 2011.

[4] S. Boyd, N. Parikh, E. Chu, B. Peleato, and J. Eckstein, "Distributed Optimization and Statistical Learning via the Alternating Direction Method of Multipliers," Foundations and Trends in Machine Learning, vol. 3, no. 1, pp. 1-122, 2011.

[5] H. Bristow, A. Eriksson, and S. Lucey, "Fast convolutional sparse coding," in IEEE Conference on Computer Vision and Pattern Recognition (CVPR), Portland, OR, USA, 2013, pp. 391-398.

[6] R. Chalasani, J. C. Principe, and N. Ramakrishnan, "A fast proximal method for convolutional sparse coding," in International Joint Conference on Neural Networks (IJCNN), Dallas, TX, USA, 2013, pp. 1-5.

[7] P. L. Combettes and H. H. Bauschke, Convex Analysis and Monotone Operator Theory in Hilbert Spaces. Springer, 2011.
[8] L. Dalcín, R. Paz, and M. Storti, "MPI for Python," Journal of Parallel and Distributed Computing, vol. 65, no. 9, pp. 1108-1115, 2005.

[9] P. del Aguila Pla and J. Jaldén, "Convolutional GroupSparse Coding and Source Localization," in IEEE International Conference on Acoustics, Speech and Signal Processing (ICASSP), 2018, pp. 2776-2780.

[10] T. Dupré la Tour, T. Moreau, M. Jas, and A. Gramfort, "Multivariate Convolutional Sparse Coding for Electromagnetic Brain Signals," in Advances in Neural Information Processing Systems (NeurIPS), Montreal, Canada, 2018, pp. 3296-3306.

[11] J. Friedman, T. Hastie, H. Höfling, and R. Tibshirani, "Pathwise coordinate optimization," The Annals of Applied Statistics, vol. 1, no. 2, pp. 302-332, 2007.

[12] D. Gabay and B. Mercier, "A dual algorithm for the solution of nonlinear variational problems via finite element approximation," Computers $\backslash \mathcal{E}$ Mathematics with Applications, vol. 2, no. 1, pp. 17-40, 1976.

[13] M. Giavalisco and the GOODS Teams, "The Great Observatories Origins Deep Survey: Initial Results From Optical and Near-Infrared Imaging," The Astrophysical Journal Letters, vol. 600, no. 2, p. L93, 2004.

[14] R. Grosse, R. Raina, H. Kwong, and A. Y. Ng, "ShiftInvariant Sparse Coding for Audio Classification," Cortex, vol. 8, p. 9, 2007.

[15] M. Jas, T. Dupré la Tour, U. Şimşekli, and A. Gramfort, "Learning the Morphology of Brain Signals Using AlphaStable Convolutional Sparse Coding," in Advances in Neural Information Processing Systems (NeurIPS), Long Beach, CA, USA, 2017, pp. 1099-1108.

[16] S. P. Karimireddy, A. Koloskova, S. U. Stich, and M. Jaggi, "Efficient Greedy Coordinate Descent for Composite Problems," in Artificial Intelligence and Statistics (AISTAT), 2019.

[17] K. Kavukcuoglu, P. Sermanet, Y.-1. Boureau, K. Gregor, and Y. Le Cun, "Learning Convolutional Feature Hierarchies for Visual Recognition," in Advances in Neural Information Processing Systems (NeurIPS), Vancouver, Canada, 2010, pp. 1090-1098.

[18] F. B. Kjolstad and M. Snir, "Ghost Cell Pattern," in Proceedings of the 2010 Workshop on Parallel Programming Patterns - ParaPLoP '10. Carefree, Arizona: ACM Press, 2010, pp. $1-9$.

[19] H. W. Kuhn, "The Hungarian method for the assignment problem," Naval Research Logistics Quarterly, vol. 2, no. 1-2, pp. 83-97, Mar. 1955.

[20] J. Mairal, F. R. Bach, J. Ponce, and G. Sapiro, “Online Learning for Matrix Factorization and Sparse Coding," Journal of Machine Learning Research (JMLR), vol. 11, no. 1, pp. 19-60, 2010.

[21] T. Moreau, L. Oudre, and N. Vayatis, "DICOD: Distributed Convolutional Sparse Coding," in International Conference on Machine Learning (ICML). Stockohlm, Sweden: PMLR (80), 2018, pp. 3626-3634.

[22] J. Nutini, M. Schmidt, I. H. Laradji, M. P. Friedlander, and H. Koepke, "Coordinate Descent Converges Faster with the Gauss-Southwell Rule Than Random Selection," in International Conference on Machine Learning (ICML), Lille, France, 2015, pp. 1632-1641.

[23] S. Osher and Y. Li, "Coordinate descent optimization for \$ \ell_1\$ minimization with application to compressed sensing; a greedy algorithm," Inverse Problems and Imaging, vol. 3, no. 3, pp. 487-503, 2009.

[24] V. Papyan, Y. Romano, M. Elad, and J. Sulam, "Convolutional Dictionary Learning via Local Processing," in IEEE 
International Conference on Computer Vision (ICCV), 2017, pp. 5306-5314.

[25] M. Pereyra and A. T. Figueiredo, "Maximum-A-Posteriori Estimation with Unknown Regularization Parameters," in European Signal Processing Conference (EUSIPCO), Nice, France, 2015, pp. 230-234.

[26] Python Software Fundation, "Python Language Reference, version 3.6," p. https:/ / python.org, 2017.

[27] D. Simon and M. Elad, "Rethinking the CSC Model for Natural Images," in Advances in Neural Information Processing Systems (NeurIPS), Vancouver, BC, Canada, 2019.

[28] E. Skau and B. Wohlberg, "A Fast Parallel Algorithm for Convolutional Sparse Coding," in IEEE Image, Video, and Multidimensional Signal Processing Workshop (IVMSP), 2018.

[29] J.-L. Starck, M. Elad, and D. L. Donoho, "Image Decomposition Via the Combination of Sparse Representation and a Variational Approach," IEEE Trans.Image Process., vol. 14, no. 10, pp. 1570-1582, 2005.

[30] J. Sulam, V. Papyan, Y. Romano, and M. Elad, "Multi-Layer Convolutional Sparse Modeling: Pursuit and Dictionary Learning," IEEE Transaction on Signal Processing, vol. 66, no. $15,2018$.

[31] J. Sulam, A. Aberdam, A. Beck, and M. Elad, “On MultiLayer Basis Pursuit, Efficient Algorithms and Convolutional Neural Networks," IEEE Transactions on Pattern Analysis and Machine Intelligence (PAMI), 2019.

[32] The ImageMagick Development Team, "ImageMagick," Jun. 2020.

[33] A. F. Vidal and M. Pereyra, "Maximum Likelihood Estimation of Regularization Parameters," in IEEE International Conference on Image Processing (ICIP). Athens, Greece: IEEE, 2018, pp. 1742-1746.

[34] B. Wohlberg, "Efficient Algorithms for Convolutional Sparse Representations," IEEE Transactions on Image Processing, vol. 25, no. 1, 2016.

[35] F. Yellin, B. D. Haeffele, and R. Vidal, "Blood cell detection and counting in holographic lens-free imaging by convolutional sparse dictionary learning and coding," in IEEE International Symposium on Biomedical Imaging (ISBI), Melbourne, Australia, 2017.

[36] Y. Zhang, H. W. Kuo, and J. Wright, "Structured local minima in sparse blind deconvolution," Advances in Neural Information Processing Systems (NeurIPS), vol. 2018-Decem, pp. 2322-2331, 2018.

[37] E. Zisselman, J. Sulam, and M. Elad, "A Local Block Coordinate Descent Algorithm for the Convolutional Sparse Coding Model," in IEEE Conference on Computer Vision and Pattern Recognition (CVPR), 2019, pp. 8208-8217.

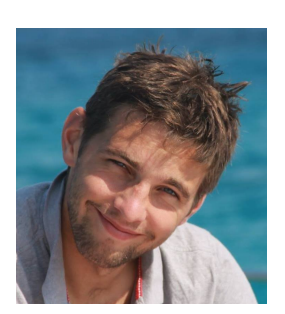

Thomas Moreau received the graduate degree from the Ecole Polytechnique, Palaiseau, France, in 2014, and the PhD degree from the Ecole Normale Supérieure, Cachan, France, in 2017 under the supervision of Nicolas Vayatis and Laurent Oudre in the CMLA laboratory. He recently joined the Inria Parietal project team in Saclay, first as a post-doctoral researcher and then as a researcher. His research interests include unsupervised learning, image/signal processing and distributed computing.

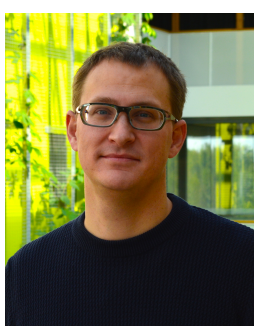

Alexandre Gramfort graduated from Ecole Polytechnique in 2005 and Telecom ParisTech in 2006, and he holds a MS in Applied Mathematics from Ecole Normale Supérieure, Cachan, France. $\mathrm{He}$ is currently senior researcher in the Parietal Team at INRIA Saclay Research Center and CEA Neurospin since 2017. He was formerly Assistant Professor at Telecom ParisTech, Université Paris-Saclay, in the image and signal processing department. His field of expertise is statistical machine learning, signal processing and scientific computing applied primarily to functional brain imaging data (EEG, MEG, fMRI). 